Effective behavior near clogging in upscaled equations for non-isothermal reactive porous media flow

Peer-reviewed author version

BRINGEDAL, Carina \& Kumar, Kundan (2017) Effective behavior near clogging in upscaled equations for non-isothermal reactive porous media flow. In: TRANSPORT IN POROUS MEDIA, 120(3), p. 553-577.

DOI: $10.1007 / \mathrm{s} 11242-017-0940-y$

Handle: http://hdl.handle.net/1942/25331 


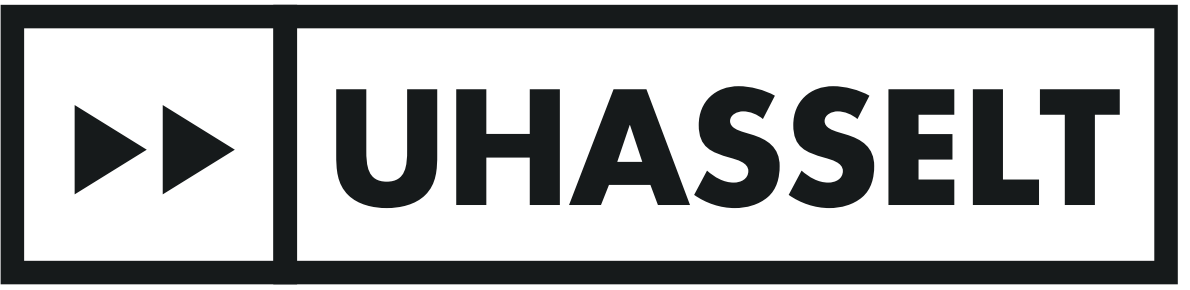

\section{Effective behavior near clogging in upscaled equations for non-isothermal reactive porous media flow}

Carina Bringedal and Kundan Kumar

UHasselt Computational Mathematics Preprint Nr. UP-17-10

October 5, 2017 


\title{
Effective behavior near clogging in upscaled equations for non-isothermal reactive porous media flow
}

\author{
Carina Bringedal • Kundan Kumar
}

Received: date / Accepted: date

\begin{abstract}
For a non-isothermal reactive flow process, effective properties such as permeability and heat conductivity change as the underlying pore structure evolves. We investigate changes of the effective properties for a two-dimensional periodic porous medium as the grain geometry changes. We consider specific grain shapes and study the evolution by solving the cell problems numerically for an upscaled model derived in Bringedal et al (2016). In particular, we focus on the limit behavior near clogging. The effective heat conductivities are compared to common porosity-weighted volume averaging approximations and we find that geometric averages perform better than arithmetic and harmonic for isotropic media, while the optimal choice for anisotropic media depends on the degree and direction of the anisotropy. An approximate analytical expression is found to perform well for the isotropic effective heat conductivity. The permeability is compared to some commonly used approaches focusing on the limiting behavior near clogging, where a fitted power law is found to behave reasonably well. The resulting macroscale equations are tested on a case where the geochemical reactions cause pore clogging and a corresponding change in the flow and transport behavior at Darcy scale. As pores clog the flow paths shift away, while heat conduction increases in regions with lower porosity.
\end{abstract}

Keywords Upscaling · Homogenization · Free boundary · Periodic porous medium · Clogging $\cdot$ Reactive flow

C. Bringedal

Geophysical Institute, University of Bergen, PO Box 7803, 5020 Bergen, Norway

Now at: Faculty of Sciences, University of Hasselt, Campus Diepenbeek, Agoralaan building

D, BE3590 Diepenbeek, Belgium

E-mail: carina.bringedal@uib.no / carina.bringedal@uhasselt.de

K. Kumar

Department of Mathematics, University of Bergen, PO Box 7803, 5020 Bergen, Norway

E-mail: kundan.kumar@uib.no

The final publication is available at Springer via https://doi.org/10.1007/s11242-017-0940-y 


\section{Introduction}

Geothermal reservoirs may encounter porosity changes induced by geochemical reactions in the pores. The injected water and the in-situ brine have different temperatures and chemical composition, triggering mineral precipitation and/or dissolution. These chemical reactions cause reservoir rock properties to develop dynamically with time due to the porosity changes. When porosity is altered the flow conditions are affected and the permeability of the medium will change. Also, as the volume contribution of fluid and rock change, effective properties such as heat conductivity is affected. For a geothermal reservoir, where subsurface heat transport and fluid flow is of high importance for the heat production performance, changes in such properties are important to model accurately to account for their possible impacts on operating conditions.

As reported from field studies and simulations, porosity and permeability changes due to precipitation and dissolution of minerals as silica, quartz, anhydrite, gypsum and calcite can occur when exploiting geothermal reservoirs (Libbey and Williams-Jones 2016; McNamara et al 2016; Mielke et al 2015; Mroczek et al 2000; Pape et al 2005; Sonnenthal et al 2005; Taron and Elsworth 2009; Wagner et al 2005; White and Mroczek 1998; Xu et al 2009). These fluid-rock interactions can alter and possibly clog flow paths, potentially affecting the performance of the geothermal plant significantly. Modeling of the mineral precipitation and dissolution is important to understand the processes and to better estimate to which extent the chemical reactions can affect the permeability and other effective properties of the porous medium. However, reactive transport affecting flow properties and heat transport can be particularly challenging to model due to the processes jointly affecting each other.

When investigating porosity and permeability changes, understanding the underlying processes at the pore scale is essential for highly coupled problems. The pore geometry affects the reaction rates as the reactive surface develops, while the permeability depends on how the geometry changes. Also, ion diffusivity and heat conductivity can be affected by the grain shape and can be anisotropic. Hence, using a Darcy scale model based on an upscaled pore scale model can give a better representation of the effective properties than common porosity-weighted average approaches. Pore scale models incorporating mineral precipitation and dissolution have been studied earlier in (van Duijn and Pop 2004; van Noorden et al 2007) and the corresponding Darcy scale models have been investigated both analytically and numerically further in (van Duijn and Knabner 1997; Knabner et al 1995; Kumar et al 2013, 2014). The rigorous derivation of upscaled model starting from the pore scale model in (van Duijn and Pop 2004) has been performed in (Kumar et al 2016) using two-scale convergence framework. These papers assume the pore geometry to be fixed, which is a valid assumption if the mineral layer is not changed much compared to the pore aperture. Upscaled pore scale models honoring porosity changes are found in (Kumar et al 2011, 2013; van Noorden $2009 a, b)$. In these papers, the position of the interface between grain and void space is tracked, giving a problem with a free boundary. Similar models can also be obtained for biofilm growth (van Noorden et al 2010), for drug release from collagen matrices (Ray et al 2013), and on an evolving microstructure (Peter 2009). These models do not include any temperature dependence in the reaction rates nor any heat transfer. 
The present work builds on the pore scale model for coupled heat transport and reactive transport in a thin strip first formulated by Bringedal et al (2015). Later this model was formulated for a periodic porous medium and upscaled to Darcy scale by Bringedal et al (2016). The freely moving interface between the mineral layer and the void space is modeled through a level set function at the pore scale. The authors derive two-dimensional effective equations honoring the pore scale dependence through cell problems. The geometry is the same as considered by van Noorden (2009a), where Bringedal et al (2016) also include heat transport as well as temperature effects in the fluid flow and in the chemical reactions. Including heat transport introduced a coupled cell problem at the pore scale with energy conservation in the void space and in the grain space, allowing for a more realistic transport of the conductive heat transfer through the pore. For geothermal systems, modeling the temperature dependences and heat transport correctly is crucial. We will build upon the upscaled model derived in (Bringedal et al 2016) by solving the cell problems and investigate the behavior of the resulting effective properties. Further, to test the model we consider an idealized case study where clogging occurs. The model by Bringedal et al (2016) does not allow phase change, but we mention that Duval et al (2004) upscaled decoupled two-phase flow with phase change using volume averaging.

The effective model in (Bringedal et al 2016) is derived for a two-dimensional domain, but Schulz et al (2016) recently derived an effective three-dimensional model for an isothermal reactive model without flow. The extension from two to three spatial dimensions could be performed by defining a tangent plane for the fluid-grain interface in the upscaling procedure, while the resulting effective model have the same appearance as in the two-dimensional case. The present paper deals only with the two-dimensional case, but a three-dimensional interpretation of the results is possible.

Our interest in this work stems from developing efficient computational techniques for deformable porous medium, in particular, in the limit case of clogging. As the upscaled equations in Bringedal et al (2016) (see Section 2) show, the Darcy scale model is coupled to cell problems (pore scale processes) via their coefficients depending upon the grain geometry. The latter is described by a level set and is in turn impacted by the Darcy scale variables such as pressure and temperature. In terms of discretization, in the upscaled two-scale model, there is a cell problem at each spatial mesh point. To obtain the effective parameters at the Darcy scale, the grain geometry at the cell problem needs to be updated at each time step and the corresponding problems need to be solved. Therefore, solving the upscaled model implies that at each time step and at each spatial mesh point, we need to solve as many cell problems as there are spatial mesh points to obtain the corresponding effective parameters. Assuming the Darcy scale domain to be a $2 \mathrm{D}$ domain, the upscaled model is a 4-dimensional model (2-dimensional for the cell problem and 2-dimensional for the Darcy scale domain). Even if these cell problems can be solved in parallel, the computational efforts involved are quite huge. Our approach here can be seen as a simplification of this full fledged approach. We assume that the geometry at the pore scale can be characterized by a single parameter and hence, the level set equation becomes an ordinary differential equation. Next, instead of solving the cell problems at each time step, we develop relationships (e.g., polynomial law) of the effective parameters based on the geometry parameter. As a result, solving the upscaled model requires simply using these fitted 
polynomials for the parameters drastically reducing the computational costs. Our approach should be contrasted with the prevalent approaches of treating porosity as an unknown, defining an ordinary differential equation for its evolution and using engineering correlations for the effective parameters such as permeability, heat conductivity, and diffusion coefficient (see e.g., (Chadam et al 1986, 1991; Zhao 2014)). In contrast to using the heuristic correlations, we use the homogenization approach to provide the polynomial fit. Moreover, even though we simplify the situation here by characterizing the geometry by only one unknown, this approach can be extended to include more parameters for characterizing the geometry and higher order fits for the effective parameters.

Our contributions in this work are in studying the evolution of effective quantities, specially near the critical porosity limit, by solving the cell problems for certain grain geometries. This work may also be interpreted as an extension of similar studies as (van Noorden 2009a) to a non-isothermal case and a focus on the near-clogging scenario. Additionally, we also provide an approximate closed form analytical solution for the cell problem and show its accuracy by comparing it to the detailed numerical simulations. In practice, there are several correlations used in engineering literature coupling porosity and effective quantities (see e.g., Verma and Pruess (1988), equation (6) below). We enrich this study by comparing some of these correlations to homogenization approach for evolving geometry. The cell problem, as obtained from the homogenization theory, is solved to provide the effective parameters for the different geometries providing us plots for the effective parameters versus the geometry. We can interpret our approach as a derivation of these correlation-type relationships. Moreover, the changes in the geometry at the pore scale may not always be symmetric. Our approach allows the flexibility of characterising the geometry in more than one variables and then studying this variation. Naturally, the more variables we take for characterising the geometry at the pore scale, we get the flexibility of describing more complex geometries but the offline computational costs increase. We also report a numerical study showing the impact of pore-scale geometry changes on the Darcy scale flow and transport.

The structure of this paper is as follows. In Section 2 we present the effective equations with corresponding cell problems, while in Section 3 we solve the cell problems numerically. Section 4 compares the numerical solution of the cell problems with analytical solution of approximate cell problems, before considering a macroscale case study with clogging in Section 5. The paper ends with a summary with some comments on applications together with some concluding remarks.

\section{Effective equations and cell problems}

The non-dimensional upscaled model by Bringedal et al (2016) considers coupled reactive flow with heat transport and varying porosity. We state here the resulting upscaled model and refer to Bringedal et al (2016) for the derivation and underlying assumptions, and references therein for justification of model choices. In the general formulation, still using a level set function to describe the pore structure, the model consists of the five (non-dimensional) unknowns $S(x, y, t), u(x, t), T(x, t)$, $\overline{\mathbf{q}}(x, t)$ and $p(x, t)$, which are the level set function, macroscopic ion concentration, temperature, flow rate and pressure, respectively. All but the first depend only on time $t$ and spatial variable $x$, which is defined for all $x=\left(x_{1}, x_{2}\right) \in \Omega, \Omega$ being 
the macroscopic; i.e., the Darcy scale, domain. The level set function $S(x, y, t)$ also depends on the microscopic variable $y=\left(y_{1}, y_{2}\right) \in\left[-\frac{1}{2}, \frac{1}{2}\right]^{2}$, where $y$ is as a zoomed-in variable resolving the pore structure at a specific macroscopic point $x \in \Omega$. The five non-dimensional upscaled equations are $(x \in \Omega, t>0)$

$$
\begin{aligned}
& \partial_{t}(\rho S(x, y, t))=f(T, u, y)\left|\nabla_{y} S(x, y, t)\right| \\
& \partial_{t}\left(\left|Y_{0}(x, t)\right| u(x, t)+\left|G_{0}(x, t)\right| \rho\right)+\nabla_{x} \cdot(\overline{\mathbf{q}}(x, t) u(x, t)) \\
& \quad=\nabla_{x} \cdot\left(D \mathcal{A}_{u}(x, t) \nabla_{x} u(x, t)\right), \\
& \partial_{t}\left(\left|Y_{0}(x, t)\right| \rho_{f}(T) T(x, t)+\left|G_{0}(x, t)\right| \varsigma \rho T(x, t)\right)+\nabla_{x} \cdot\left(\rho_{f}(T) \overline{\mathbf{q}}(x, t) T(x, t)\right) \\
& \quad=\nabla_{x} \cdot\left(\kappa_{f} \mathcal{A}_{f}(x, t) \nabla_{x} T(x, t)+\kappa_{g} \mathcal{A}_{g}(x, t) \nabla_{x} T(x, t)\right), \\
& \partial_{t}\left(\left|Y_{0}(x, t)\right| \rho_{f}(T)+\left|G_{0}(x, t)\right| 2 \rho\right)+\nabla_{x} \cdot\left(\rho_{f}(T) \overline{\mathbf{q}}(x, t)\right)=0, \\
& \overline{\mathbf{q}}(x, t)=-\frac{1}{\mu_{f}(T)} \mathcal{K}(x, t) \nabla_{x} p(x, t) .
\end{aligned}
$$

In the above equations, $\rho$ is the constant molecular density of the mineral, while $\rho_{f}$ and $\mu_{f}$ are the molecular density and viscosity of the fluid and are allowed to depend on temperature. Further, $D$ is the molecular diffusivity of the fluid, while $\kappa_{f}$ and $\kappa_{g}$ are the heat conductivities of fluid and grain, respectively. These are considered properties of the fluid/grain and are constant. The reaction rate $f$ describes the mineral precipitation and dissolution and is given by

$$
f(T, u, y)=k e^{-\alpha / T}\left(\frac{u^{2}}{K_{m}(T)}-w(\operatorname{dist}(y, B), T, u)\right)
$$

where $k$ is the Damköhler number, and $\alpha=E / R T_{\text {ref }}$ where $E$ is the activation energy, $R$ the gas constant and $T_{r e f}$ a reference temperature. Further, $K_{m}(T)$ is the solubility of the mineral in question, and the function $w(\operatorname{dist}(y, B), T, u)$ is defined by

$$
w(d, T, u)= \begin{cases}0 & \text { if } d<0 \\ \min \left(\frac{u^{2}}{K_{m}(T)}, 1\right) & \text { if } d=0 \\ 1 & \text { if } d>0\end{cases}
$$

where the distance corresponds to the width of the mineral layer. The spaces $G_{0}(x, t), Y_{0}(x, t)$ and $\Gamma_{0}(x, t)$ refers to the microscopic grain space, void space and the interface between them, inside the unit cell $y \in\left[-\frac{1}{2}, \frac{1}{2}\right]^{2}$, see Figure 1 . These spaces are defined implicitly as where the level set function is positive, negative and zero, respectively. The notation $|\cdot|$ refers to the area (volume) of the space. The effective coefficients $\mathcal{A}_{u}, \mathcal{A}_{f}, \mathcal{A}_{g}$ and $\mathcal{K}$ represents the porous medium's ability to transmit ions through diffusion, heat through conduction in fluid/grain, and fluid 
through fluid flow. The effective coefficients are tensors with components

$$
\begin{aligned}
& a_{i j}^{u}(x, t)=\int_{Y_{0}(x, t)}\left(\delta_{i j}+\partial_{y_{i}} v^{j}(y)\right) d y, \\
& a_{i j}^{f}(x, t)=\int_{Y_{0}(x, t)}\left(\delta_{i j}+\partial_{y_{i}} \Theta_{f}^{j}(y)\right) d y, \\
& a_{i j}^{g}(x, t)=\int_{G_{0}(x, t)}\left(\delta_{i j}+\partial_{y_{i}} \Theta_{g}^{j}(y)\right) d y, \\
& k_{i j}(x, t)=\int_{Y_{0}(x, t)} \omega_{i}^{j}(y) d y,
\end{aligned}
$$

respectively, for $i, j=1,2$. The functions $v^{j}(y), \Theta_{f}^{j}(y), \Theta_{g}^{j}(y)$ and $\omega^{j}(y)$ are solutions of the cell problems $\left(x \in \Omega, y \in\left[-\frac{1}{2}, \frac{1}{2}\right]^{2}, t>0\right)$

$$
\begin{gathered}
\nabla_{y}^{2} v^{j}(y)=0 \text { in } Y_{0}(x, t) \text { and } \mathbf{n} \cdot\left(\mathbf{e}_{j}+\nabla_{y} v^{j}(y)\right)=0 \text { at } \Gamma_{0}(x, t), \\
\nabla_{y}^{2} \Theta_{f}^{j}(y)=0 \text { in } Y_{0}(x, t) \text { and } \nabla_{y}^{2} \Theta_{g}^{j}(y)=0 \text { in } G_{0}(x, t) \text { where } \\
\kappa_{f} \mathbf{n}_{0} \cdot\left(\mathbf{e}_{j}+\nabla_{y} \Theta_{f}^{j}(y)\right)=\kappa_{g} \mathbf{n}_{0} \cdot\left(\mathbf{e}_{j}+\nabla_{y} \Theta_{g}^{j}(y)\right) \\
\text { and } \Theta_{f}^{j}(y)=\Theta_{g}^{j}(y) \text { at } \Gamma_{0}(x, t), \\
\mathbf{e}_{j}+\nabla_{y} \Pi^{j}(y)=-\nabla_{y}^{2} \omega^{j}(y) \text { and } \nabla_{y} \cdot \omega^{j}(y)=0 \text { in } Y_{0}(x, t) \\
\text { and } \omega^{j}(y)=0 \text { at } \Gamma_{0}(x, t)
\end{gathered}
$$

together with the periodicity in $y$ for $v^{j}(y), \Theta_{f}^{j}(y), \Pi^{j}(y)$ and $\omega^{j}(y), j=1,2$. The periodicity assumption comes from the macroscopic domain being filled with microscopic cells, as the one seen in Figure 1, lying adjacent to each other. Hence, the periodic boundary condition is due to the proximity of the neighboring cells. However, the neighboring cells do not have to be equal; hence inhomogeneities are allowed.

We will consider a simplified version of this formulation by imposing constraints on the pore space geometry. By assuming the grains to have a specific shape described through a single variable, the level set equation can be replaced by an equation for that variable. Here, we show how the model equations change when using circular grains, but the approach is more general and can be used as long the grain shape can be described through one free parameter. For circular grains we introduce the level set function $S(x, y, t)=R^{2}(x, t)-y_{1}^{2}-y_{2}^{2}$, where $R(x, t)$ is the radius of the grains. Note that the grain radius is a function of position (and time) as the grain radius can vary between unit cells, allowing non-homogeneous media. The upscaled equations will depend on grain radius $R(x, t)$ instead of the level set function. This simplification was also made by van Noorden (2009a), but places severe constraints on the pore structure and should be interpreted as a choice made for visualization purposes. We mention that Frank et al (2011); Frank (2013) implemented other choices of the level set function for a StokesNernst-Planck-Poisson system, and in the following section we also solve the cell problems for elliptic grains to indicate the effect of anisotropy. Using the circular 


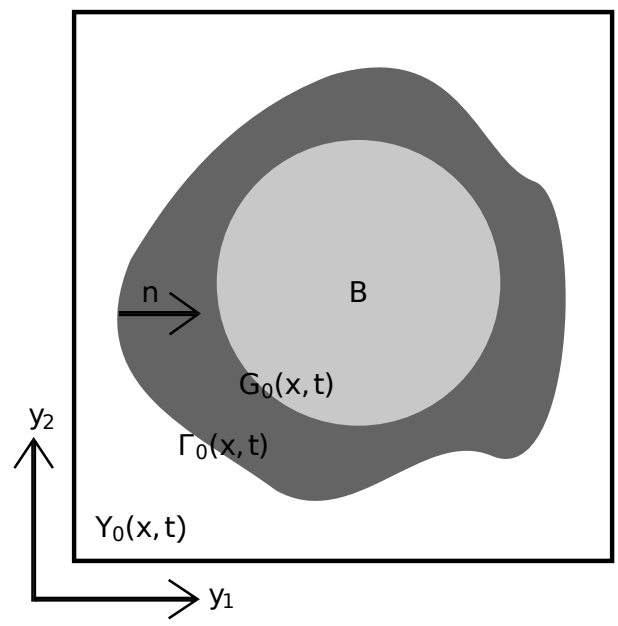

Fig. 1 Model of microscopic pore, with fluid space $Y_{0}(x, t)$, grain space $G_{0}(x, t)$ and the interface between them $\Gamma_{0}(x, t)$, which has unit normal $\mathbf{n}$ pointing into the grain. Note that the grain consists of both a reactive mineral layer (dark grey) surrounding a non-reactive part $B$ (light grey).

geometry the Darcy scale model equations now becomes, for $(x \in \Omega, t>0)$

$$
\begin{aligned}
& \partial_{t}(\rho R(x, t))=f(T, u, R) \\
& \partial_{t}\left(\left(1-\pi R^{2}(x, t)\right) u(x, t)+\pi R^{2}(x, t) \rho\right)+\nabla_{x} \cdot(\overline{\mathbf{q}}(x, t) u(x, t)) \\
& \quad=\nabla_{x} \cdot\left(D \mathcal{A}_{u}(R) \nabla_{x} u(x, t)\right) \\
& \partial_{t}\left(\left(1-\pi R^{2}(x, t)\right) \rho_{f}(T) T(x, t)+\pi R^{2}(x, t) \varsigma \rho T(x, t)\right)+\nabla_{x} \cdot\left(\rho_{f}(T) \overline{\mathbf{q}}(x, t) T(x, t)\right) \\
& \quad=\nabla_{x} \cdot\left(\kappa_{f} \mathcal{A}_{f}(R) \nabla_{x} T(x, t)+\kappa_{g} \mathcal{A}_{g}(R) \nabla_{x} T(x, t)\right) \\
& \partial_{t}\left(\left(1-\pi R^{2}(x, t)\right) \rho_{f}(T)+\pi R^{2}(x, t) 2 \rho\right)+\nabla_{x} \cdot\left(\rho_{f}(T) \overline{\mathbf{q}}(x, t)\right)=0 \\
& \overline{\mathbf{q}}(x, t)=-\frac{1}{\mu_{f}(T)} \mathcal{K}(R) \nabla_{x} p(x, t) .
\end{aligned}
$$

The reaction rate $f$ uses the distance between $R$ and $R_{\min }$, where $R_{\min }$ is the radius of the nonreactive solid, to calculate the width of the mineral layer. The matrices $\mathcal{A}_{u}, \mathcal{A}_{f}, \mathcal{A}_{g}$ and $\mathcal{K}$ depend only on grain radius $R(x, t)$ as the integration area is determined by the radius alone. The cell problems are defined as before, but where the unit cell spaces are now defined using the grain radius. Hence, the pore space is $Y_{0}(x, t)=\left\{y \in\left[-\frac{1}{2}, \frac{1}{2}\right]^{2} \mid y_{1}^{2}+y_{2}^{2}>R^{2}(x, t)\right\}$, grain space is $G_{0}(x, t)=\left\{y \in\left[-\frac{1}{2}, \frac{1}{2}\right]^{2} \mid y_{1}^{2}+y_{2}^{2}<R^{2}(x, t)\right\}$ and the interface between them is $\Gamma_{0}(x, t)=\left\{y \in\left[-\frac{1}{2}, \frac{1}{2}\right]^{2} \mid y_{1}^{2}+y_{2}^{2}=R^{2}(x, t)\right\}$. The tensors $\mathcal{A}_{u}, \mathcal{A}_{f}, \mathcal{A}_{g}$ and $\mathcal{K}$ defined in equation (2) will now be cheaper to compute, and are due to the symmetric grain shape scalars. 


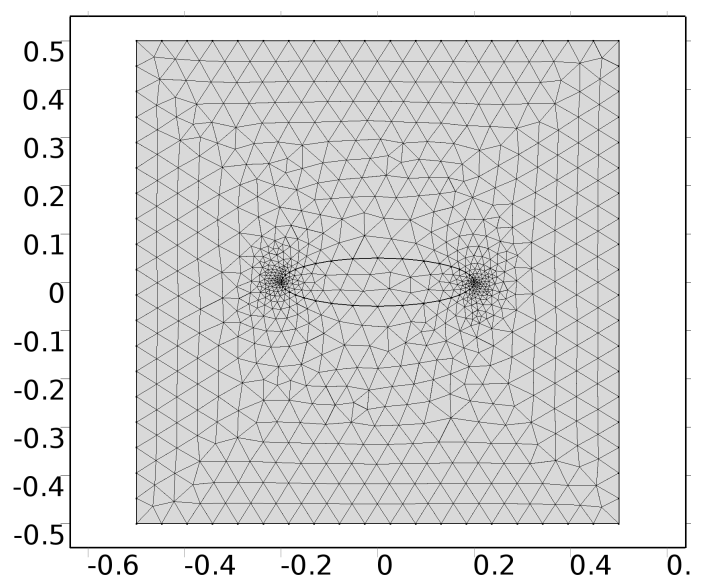

Fig. 2 Mesh of a microscopic pore geometry with elliptic grain space with major axis 0.4 and minor axis of 0.1 (contrast of 4). Also, see Figure 1 showing a representative unit cell geometry and Figure 7 showing an elliptic grain geometry.

\section{Numerical solution of cell problems}

The cell problems are decoupled from the macroscale equations and solved beforehand. For various fixed values of grain radius $R$ the cell problems (3) can be solved, hence providing effective quantities $\mathcal{A}_{u}, \mathcal{A}_{f}, \mathcal{A}_{g}$ and $\mathcal{K}$ that are functions of $R$. Note that the cell problems are second order linear elliptic problems. The permeability and diffusion tensors cell problems are defined in the pore space with appropriate boundary conditions on the grain geometry whereas the thermal problem is defined in the whole cell geometry together with interface conditions across the grain-pore space boundary. To solve these elliptic problems, we adopt standard approaches. We write down a weak formulation for these cell problems and use standard finite element techniques as implemented in the Finite Element package COMSOL multiphysics (COMSOL Inc. 2011). The coupled cell problems for effective heat conductivity coefficients $\mathcal{A}_{f}$ and $\mathcal{A}_{g}$ have not been considered earlier. We will in this paper focus on circular grains, which represents an isotropic medium. However, in this section we also solve the cell problems for elliptic grains to sketch the effect of anisotropy on the effective quantities. For solving these cell problems, we use Finite Element Method with triangular elements using P1-basis functions. Since we consider a sequence of pore scale geometry, the corresponding mesh also changes. For example, as shown in Figure 2, for the case of thermal conductivity computations, elliptic grain shape, with major axis diameter 0.4 and minor axis diameter of 0.1 (contrast of 4), the number of triangular elements is 1602 . We use the automatic meshing and solution approach as implemented in COMSOL Inc. (2011). The post-processing of the solution to obtain the effective quantities is also performed using the available tools there. Note that the effective quantities are all non-dimensional according to the non-dimensionalization in Bringedal et al (2016). 

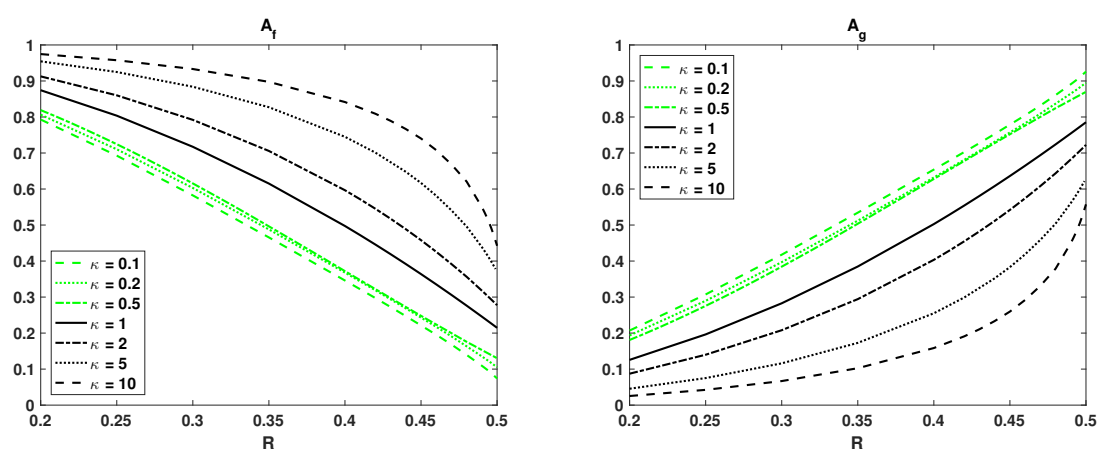

Fig. 3 Effective heat conductivity coefficients for fluid (left) and grain (right). Increasing grain radius $R$ corresponds to less void space. When $\kappa$ is larger than 1 , the grain heat conductivity is larger than in the fluid.

\subsection{Circular grains}

As the resulting effective quantities $\mathcal{A}_{u}, \mathcal{A}_{f}, \mathcal{A}_{g}$ and $\mathcal{K}$ are known to be scalars when the grains are circular due to isotropy, it is only necessary to solve the equations $(2,3)$ for $i=j=1$. The resulting effective diffusion coefficient $\mathcal{A}_{u}$ is identical as the one considered by (van Noorden 2009a) and the results are not presented here.

The heat conductivity coefficients can be interpreted as a weighting of the relative importance of the void space and grain space, as a more accurate alternative to the usual porosity-weighting since the actual heat transfer within and between fluid and grain is taken into account. The sum of $\mathcal{A}_{f}$ and $\mathcal{A}_{g}$ is always 1 . Figure 3 shows the effective heat conductivity coefficients as a function of grain radius $R$, for various values of $\kappa=\kappa_{g} / \kappa_{f}$. The case $\kappa=1$ corresponds to when the heat conductivities in fluid and grain are equal. In this case, the cell problem is trivial and $\mathcal{A}_{f}=\phi=1-\pi R^{2}$ and $\mathcal{A}_{g}=1-\phi=\pi R^{2}$, which corresponds to the usual porosity weighting. The figures only display values for radii between 0.2 and 0.5 . A radius of 0.5 corresponds to the porous medium being clogged (although there is still void space caught between the circular grains), while we have not considered radii less than $R_{\min }=0.2$ even though the cell problems are well-defined for radii down to 0.

Figure 4 shows the effective heat conductivity of the medium that is defined as

$$
\kappa_{\mathrm{eff}}=\kappa_{f} \mathcal{A}_{f}+\kappa_{g} \mathcal{A}_{g}
$$

In Figure $4 \kappa_{f}=1$, hence the value of $\kappa=\kappa_{g} / \kappa_{f}$ corresponds to the value of $\kappa_{g}$. Hence, all lines approach 1 if extending the plot to $R=0$. Figure 5 compares two of the effective heat conductivities with the usual volume averaged heat conductivities

$$
\begin{aligned}
\kappa_{\mathrm{eff}, \mathrm{ar}} & =\kappa_{f}\left(1-\pi R^{2}\right)+\kappa_{g} \pi R^{2}, \\
\kappa_{\mathrm{eff}, \mathrm{geo}} & =\kappa_{f}^{\left(1-\pi R^{2}\right)} \kappa_{g}^{\left(\pi R^{2}\right)}, \\
\kappa_{\mathrm{eff}, \text { har }} & =\frac{1}{\frac{1-\pi R^{2}}{\kappa_{f}}+\frac{\pi R^{2}}{\kappa_{g}}},
\end{aligned}
$$




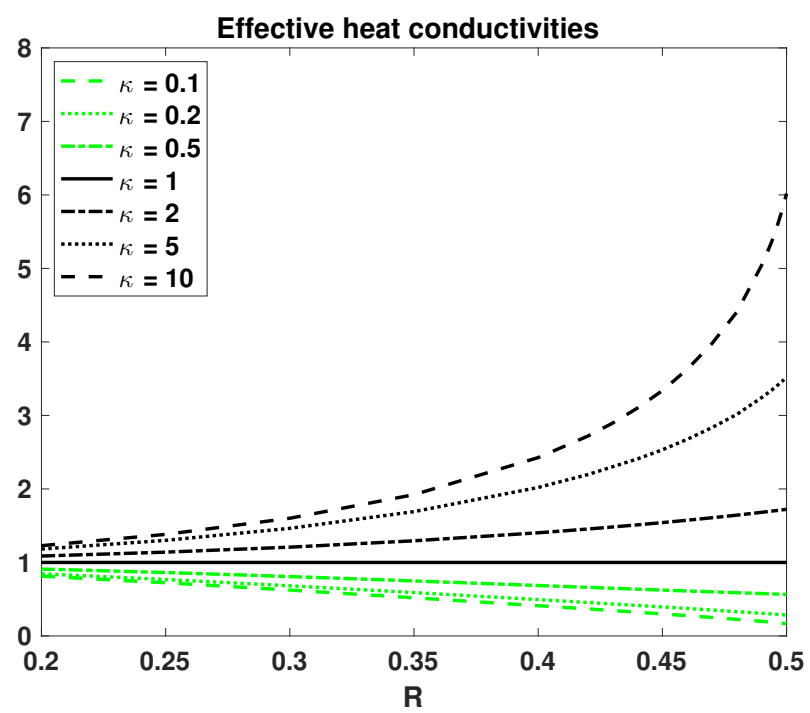

Fig. 4 Effective heat conductivities for the porous medium. In all cases, $\kappa_{f}=1$, hence $\kappa$ reflects the value of the heat conductivity in the grain $\kappa_{g}$.

which are the arithmetic, geometric and harmonic averages, respectively. As seen in the figure, the effective heat conductivities calculated from the cell problems always give a smaller value than the arithmetic averaged value, which is the one usually employed for porous media (Nield and Bejan 2013). The overestimation of the effective heat conductivity by the arithmetic averaging can be understood as following: Consider a case where the heat conductivity is much larger in grain than in fluid, corresponding to an extreme case of $\kappa=2$ shown in Figure 5. The unit cell would still experience a relatively low heat conductivity for low grain radii, as the grains are isolated from each other by the low-conductive fluid. However, when the grain radius is so high that the medium almost clog and the distance between the highly conductive grains is smaller, the heat conductivity will increase substantially. This behavior is captured by the cell problem solutions, as illustrated by the line for $\kappa=10$ in Figure 4, while an arithmetic averaging based on porosity will not be able to capture such behavior and instead overestimates the importance of the highly conductive phase. The cell problem solution is best approximated by the geometric mean, which is the known as the more suitable solution for random porous media (Woodside and Messmer 1961). Also for such an extreme case of $\kappa=10$, the geometric mean is the best choice (not shown). The case with $\kappa_{f}=\kappa_{g}$ is trivial and gives equal results for all methods.

The permeability $\mathcal{K}$ is identical as the one found by van Noorden (2009a), and is displayed in Figure 6. As grain radii close to 0.5 corresponds to being close to clogging, the permeability quickly approaches zero for growing grain radius. From the logarithmic plot of the permeability, we can estimate that the permeability approaches zero as the grain radius approaches 0.5 with an inclination number corresponding to approximately $O\left((R-0.5)^{5 / 2}\right)$. 


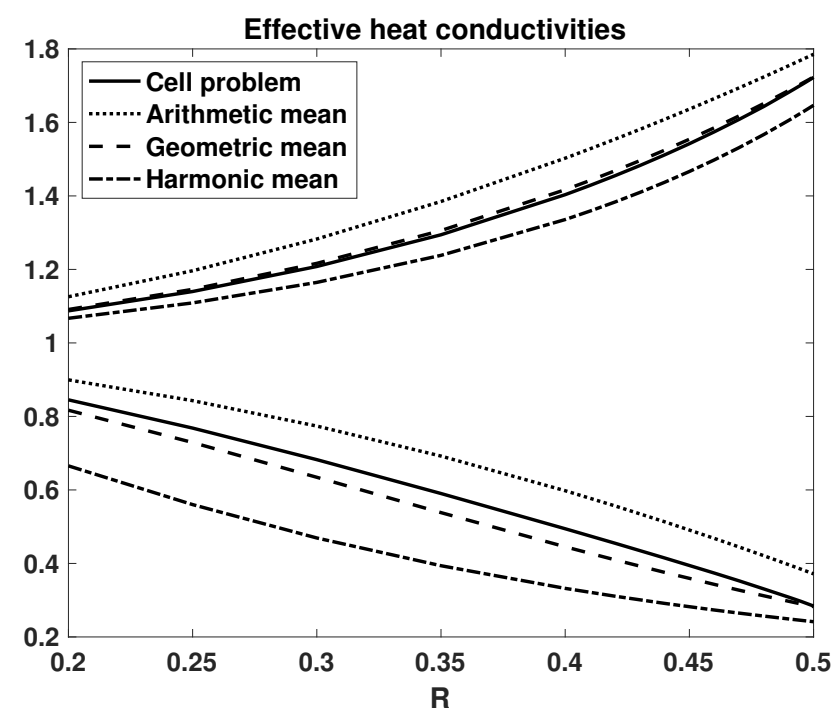

Fig. 5 Effective heat conductivities for the porous medium. The solid curves arise from the cell problem formulation, the dotted are the arithmetic means, the dashed are the geometric means and the dashed-dotted are the harmonic means. The four top curves are for $\kappa=2$, while the four lower are for $\kappa=0.2$.
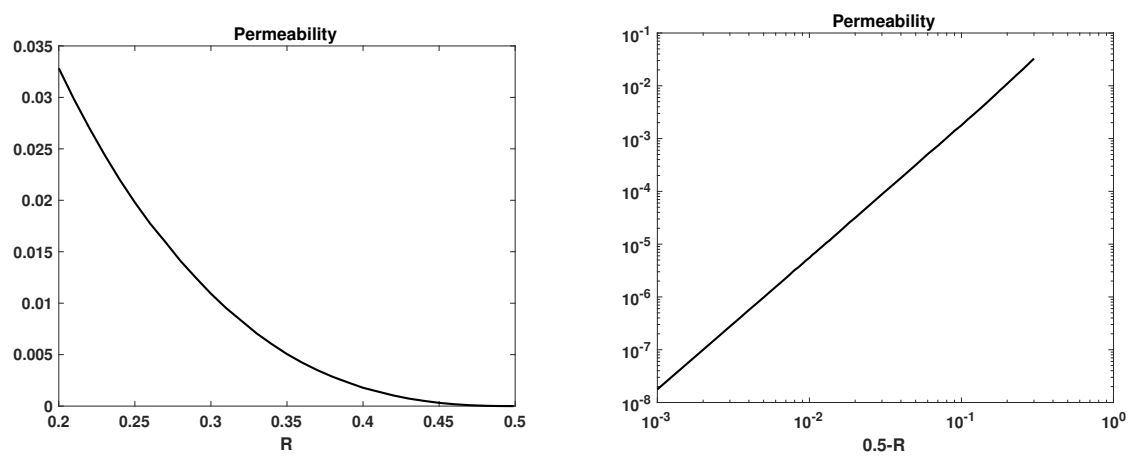

Fig. 6 Permeability of the porous medium. The right plot shows a log-log plot for the permeability as a function of $(0.5-R)$, as the permeability approaches zero when the grain radius approaches 0.5 . Note that the permeability values are non-dimensional, but dimensional $\left(\mathrm{m}^{2}\right)$ values can be obtained by scaling with $l^{2}$, where $l$ is the typical pore size; i.e., the scaling such that the cell in Figure 1 has side lengths 1.

\subsection{Elliptic grains}

The level set formulation can also be applied to investigate other grain shapes than the isotropic circular shape, and we mention here the differences when going into an anisotropic regime using elliptic grains. We assume the grain in each unit cell being elliptic with a fixed ratio between the semi-major and semi-minor axis. When the grain grows and shrinks due to mineral precipitation and dissolution, the grain would not preserve its elliptic shape due to the underlying assumption 


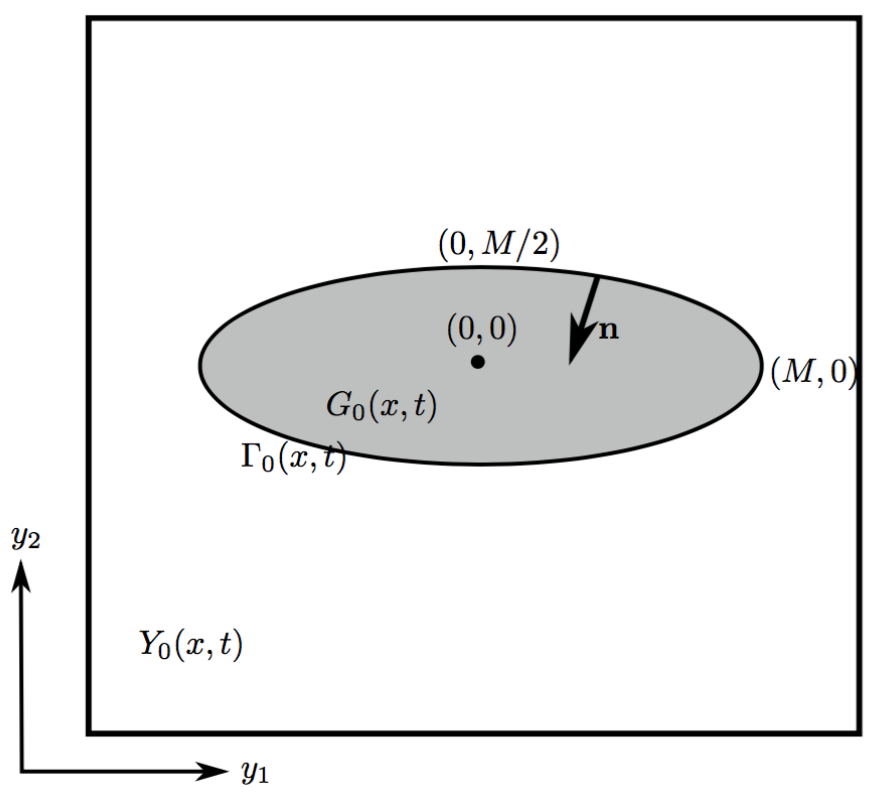

Fig. 7 Model of microscopic pore with elliptic shape with ratio of 2 between the semi-major and semi-minor axis. The unit cell has fluid space $Y_{0}(x, t)$, grain space $G_{0}(x, t)$ and the interface between them $\Gamma_{0}(x, t)$, which has unit normal $\mathbf{n}$ pointing into the grain.

of even growth/dissolution arising from the upscaling and derivation of the cell problem (Bringedal et al 2016). However, making physical arguments through the grain shape itself locally allowing for higher or lower reaction rates, we could argue that the elliptic shape should be maintained, hence the shape of the grain can at all times be explained through the parameter $M$; the length of the semi-major axis. See Figure 7 for a sketch of the unit cell, where the ratio between the semi-major and semi-minor axis of the grain is 2 .

The formulation of the upscaled equations would be similar as in equations (4), replacing the radius $R(x, t)$ with the semi-major axis $M(x, t)$, and taking into account the fluid volumes and grain volumes changing. Now, $\left|Y_{0}(x, t)\right|=1-\pi M^{2} / 2$ and $\left|G_{0}(x, t)\right|=\pi M^{2} / 2$ when the ratio between semi-major and semi-minor axis is 2. As earlier, we assume there is some part of the grain that will not dissolve and choose this to be for $M_{\min }=0.2$. This minimum choice of $M$ corresponds to a different porosity than $R_{\min }=0.2$. The maximum semi-major axis is $M=0.5$, corresponding to clogging. Note however that $M=0.5$ resembles a layered medium with no flow in the $y_{2}$-direction, but still allowing flow in the $y_{1}$-direction. The porosity is significantly higher than in the circular case with $R=0.5$.

Due to the anisotropy of the grain shape the resulting effective permeability, diffusivity and heat conductivities in equation (2) will no longer be scalars, but $2 \times 2$ matrices. The cell problems (3) are solved using elliptic grain shape with $M \in[0.2,0.5)$ using COMSOL multiphysics (COMSOL Inc. 2011). For the heat conductivities, we only consider the two cases $\kappa=0.2$ and $\kappa=2$ as our goal is only to sketch the effect of anisotropy arising from the cell problem geometry. Although 

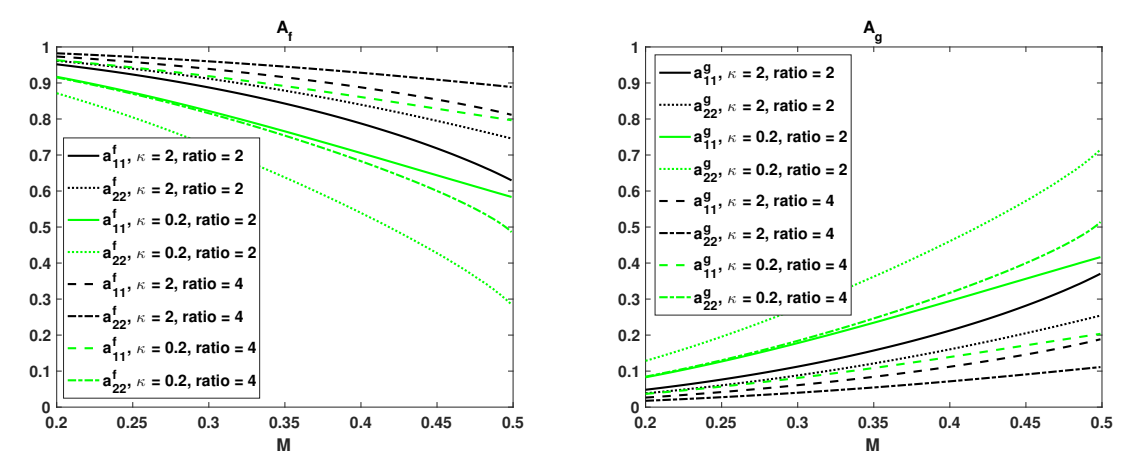

Fig. 8 Comparison of the effective heat conductivity coefficients for fluid (left) and grain (right). Increasing $M$ corresponds to more grain space, while 'ratio' refers to the ratio between semi-major and semi-minor axis of the elliptic grain. When $\kappa$ is larger than 1 (black lines), the grain heat conductivity is larger than in the fluid. Oppositely for $\kappa$ smaller than 1 (green lines).

the cell problems provide $2 \times 2$ matrices, the off-diagonal terms are effectively zero due to the orientation of the grain shape; the anisotropy is aligned with the grid. Hence, we only focus on the diagonal terms. These diagonal values are also the eigenvalues of the matrices, which are important for characterizing the anisotropic medium.

Figure 8 shows the heat conductivity coefficients for the fluid and grain as a function of semi-major axis $M$, using $\kappa=\kappa_{g} / \kappa_{f}$ either equal to 0.2 or 2 , and ratio between the semi-major and semi-minor axis being either 2 or 4 . These plots should be compared to Figure 3 for circular grains. There are several interesting findings for the anisotropic heat conductivities. Firstly, the differences between the $y_{1}$ - and $y_{2}$-coefficients are significantly different already for a relatively small ratio between the semi-major and semi-minor axis, which becomes more visible when the grain is larger (corresponding to the anisotropic feature becoming more relevant), and these differences are important both for when heat conductivity is largest in the fluid or in the grain. Whether the heat conductivity coefficient is largest in the $y_{1}$ - or $y_{2}$-direction depends on the value of $\kappa$, and phase: When $\kappa=2$, the horizontally elongated shape of the grain makes the grain heat conductivity coefficient larger in the $y_{1}$-direction than in the $y_{2}$-direction, and oppositely for the fluid. Hence, the contribution from grain on heat conduction is more important for the $y_{1}$-direction than in $y_{2}$ in this case, contributing to a larger medium heat conductivity in the $y_{1}$-direction as the grain heat conductivity is larger than in the fluid. From Figure 8 we see how the grain heat conductivity coefficient is larger in the $y_{2}$-direction than in the $y_{1}$-direction when $\kappa=0.2$. Hence, the grain heat conductivity gives a larger to contribution to the effective heat conductivity in the $y_{2}$-direction than in the $y_{1}$-direction. However, this contribution is a "negative" one as the heat conductivity in the grain is smaller than in fluid when $\kappa=0.2$. Hence, the grain conductivity hampers the medium heat conductivity to a larger extent across the layers. The effective heat conductivities seen in Figure 9 show how the heat conductivity is always larger in $y_{1}$-direction for both values of $\kappa$. Hence, heat conductivity of the medium is always more efficient along the layers than across, independently of whether the fluid or grain has the largest conductivity. 

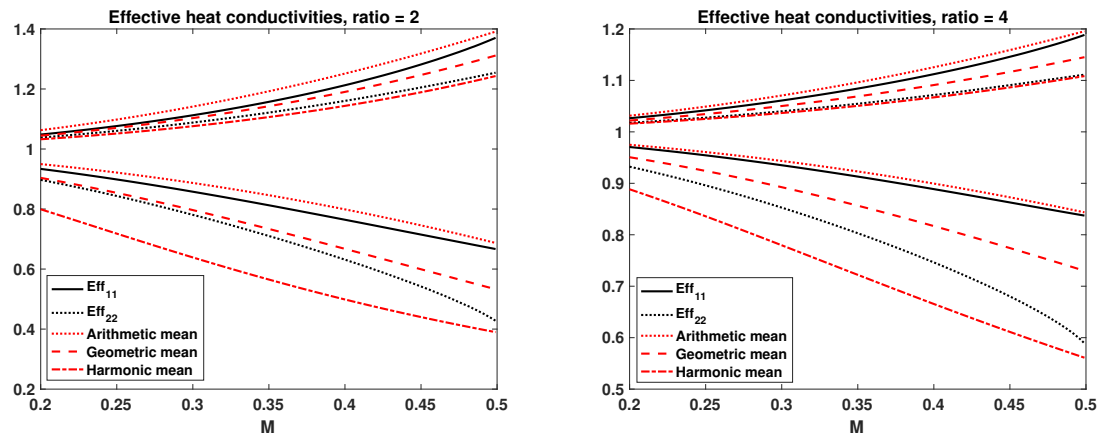

Fig. 9 Comparison of the effective heat conductivities for the porous medium for when the ratio between semi-major and semi-minor axis is 2 (left) and 4 (right). The five top curves are for $\kappa=2$, while the five lower are for $\kappa=0.2$.

Comparing the effective heat conductivities with corresponding arithmetic, geometric and harmonic averages based on (5), reveals some different behavior for the elliptic case as seen for the circular grains in Figure 5. Figure 9 shows how the different effective behaviors in the $y_{1}$ - and $y_{2}$-directions are approximated by the arithmetic, geometric and harmonic averages for the two choices of $\kappa$. While the circular grain effective heat conductivities were always closer to the geometric mean, the effective heat conductivity in the $y_{1}$-direction (along layering) is best approximated by the arithmetic mean (low $\kappa$ ) or by the geometric mean (high $\kappa$ and low $M$ ), and the effective heat conductivity in the $y_{2}$-direction (across layering) is best approximated by the harmonic mean (high $\kappa$ ) or by the geometric mean (low $\kappa$ and low $M$ ). These findings were to some extent expected as harmonic mean is known to be more suitable for series distributions and arithmetic mean for parallel distributions (Woodside and Messmer 1961). However, as seen from Figure 9, the geometric averages can still be the best alternative for lower values of $M$ and especially for small ratio between the semi-major and semi-minor axis, corresponding to when the anisotropic effect is not so strong. Hence, using arithmetic mean along the layering and harmonic mean across the layering is important when there are strong anisotropic effects in the porous medium.

For the ion diffusivity, the question of which type of averaging is not so relevant as the ion diffusivity in the grain is zero, hence only the arithmetic mean would be applicable. In practice, the resulting diffusivity coefficient is then the porosity, which is often used when modeling diffusivities with varying porosity. However, as Figure 10 shows, the porosity is a poor approximation for the effective diffusivity coefficient, even for circular geometries. The porosity approximates the diffusivity in the $y_{1}$-direction (along layers) quite well, especially when anisotropic effects are strong, but gives a poor approximation for the $y_{2}$-direction (across layers), which is zero when $M$ is 0.5 due to clogging in the $y_{2}$-direction, while the porosity is still large.

The permeability is also a tensor when considering elliptic grain shapes. The off-diagonal terms are effectively zero due to the orientation of the grain, hence we only need to consider the two diagonal terms. Here, the $y_{2}$-term will approach zero when $M$ approaches 0.5 due to clogging, while the $y_{1}$-term will still have a large permeability when $M$ is 0.5 . Figure 11 shows the diagonal components of 


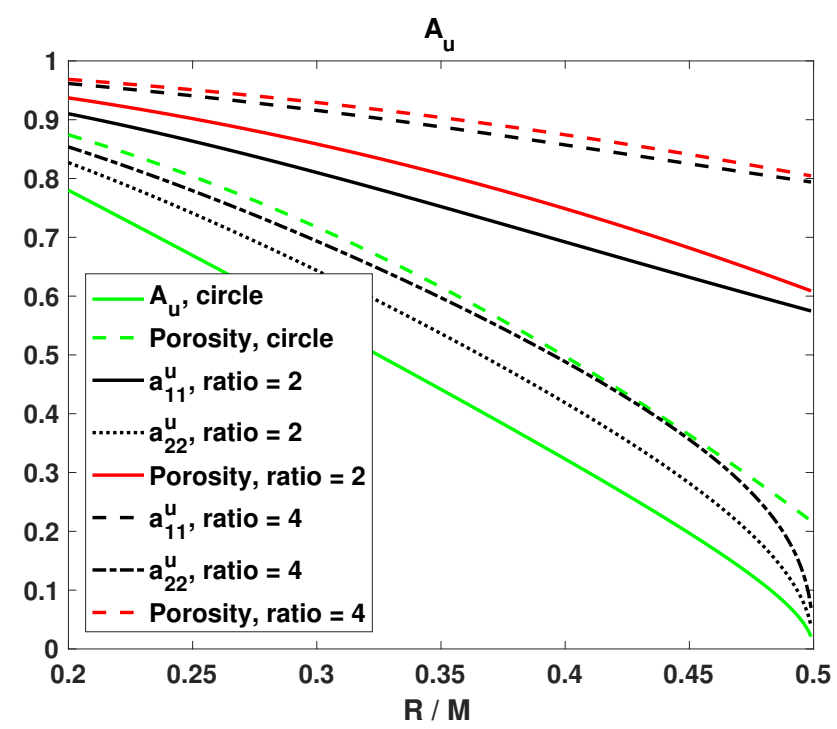

Fig. 10 Comparison of the effective ion diffusivity coefficients for circular and elliptic grains, as a function of either grain radius $R$ or the semi-major axis $M$. Increasing $R$ or $M$ corresponds to more grain space, while 'ratio' refers to the ratio between semi-major and semi-minor axis of the elliptic grain.
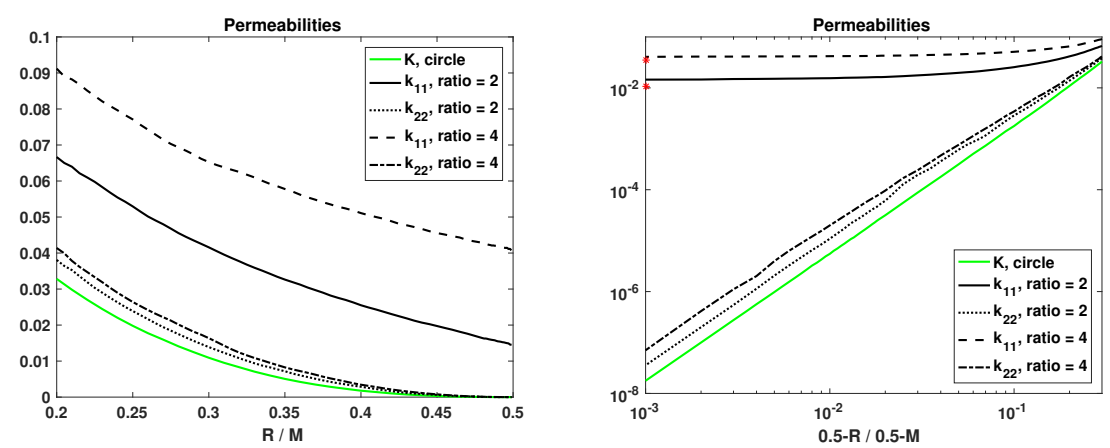

Fig. 11 Comparison of the permeabilities for the porous medium for circular and elliptic grains, as a function of either grain radius $R$ or the semi-major axis $M$. The right plot shows a log-log plot of the permeabilities as a function of $(0.5-R)$ or $(0.5-M)$, as clogging occurs when $R$ or $M$ approaches 0.5 . A ratio of 4 means that the grain is more elongated. The red stars in the right plot indicates the estimated permeability values based on an upscaled thin strip model (Bringedal et al 2015).

the permeability tensor for the elliptic grain as function of the semi-major axis $M$. For comparison, the circular grain permeability is also included.

As seen from Figure 11, the permeabilities in the $y_{1}$-direction does not approach zero when $M$ approaches 0.5 . Due to the elliptic grain shape, the medium is not blocked in the $y_{1}$-direction but forms channels. For channels, it is shown earlier (Bringedal et al 2015) that the non-dimensional permeability should behave as $K=d^{3} / 12$ where $d$ is the aperture. If we use the minimum distance between two 
adjacent grains as a measure of the aperture in the $y_{1}$-direction of the channellike medium, the cubic relationship estimates a (non-dimensional) permeability of $K=0.0105$ and $K=0.0352$ for when the ratio between the semi-major and semiminor axis are 2 and 4 , respectively. These two values are marked with red stars in the right part of Figure 11. The $k_{11}$ permeability values calculated from the cell problems when $M$ is close to 0.5 , are $k_{11}=0.0145$ and $k_{11}=0.0406$ for these two ratios. Hence, the cubic relationship slightly underestimates the calculated alongchannel permeabilities, which is possibly due to the channel not being straight but is generally wider than the minimum value used here. Also, the cell problems are not solved for $M=0.5$, as the flow cell problem is undefined in this case, hence the comparison is made for $M=0.499$.

The circular grain permeability and elliptic grain $k_{22}$-permeabilities all approach 0 when $R$ or $M$ approach 0.5 , although at slightly different speeds. Due to the anisotropy the clogging happens at different critical porosities. The critical porosity is defined as the remaining porosity when clogging (in the $y_{2}$-direction) occurs and will be $\phi_{\text {crit }}=1-\pi 0.5^{2}$ for the circular grain, and $\phi_{\text {crit }}=1-\pi 0.5^{2} / 2$ and $\phi_{\text {crit }}=1-\pi 0.5^{2} / 4$ for the elliptic grain for ratio equal to 2 and 4 , respectively. TOUGHREACT (Xu et al 2012) incorporates a power-law for the permeability based on Verma and Pruess (1988) for when there is a known critical porosity, namely

$$
K=K_{0}\left(\frac{\phi-\phi_{\text {crit }}}{\phi_{0}-\phi_{\text {crit }}}\right)^{n}
$$

where $K\left(\phi_{0}\right)=K_{0}$ at some other porosity. $\phi_{0}>\phi_{\text {crit }}$ The power $n$ is, as the critical porosity, medium dependent and needs to be estimated. Using the permeabilities at $R_{\min }, M_{\min }=0.2$ and the known critical porosities, we can estimate $n$ through a least-square approach. We find $n=2.82$ for the circular grain, while $n=2.68$ and $n=2.53$ are the powers best estimating the elliptic grain cases for when the ratio between semi-major and semi-minor axis is 2 and 4 , respectively. See Figure 12 for illustration of these three cases. Due to fitting of the entire interval, the resulting approximated permeabilities are slightly too steep when approaching the critical porosities, but do a fairly good job in capturing the behavior of the permeability near clogging.

\section{Comparison with approximate solutions}

The cell problems (3) can be solved numerically for discrete values of grain radius $R$, but when implementing the macroscale equations (4), it is more efficient if the effective quantities can be known through analytical expressions instead of solving the cell problems for each time step as the radius will vary with both time and space. One way to get around this is to solve the cell problems beforehand for a large number of discrete $R$ values and create a lookup-table, but we here investigate the possibility of approximating the cell problem solutions with either solving a related cell problem analytically, or by solving the cell problems numerically for discrete values of $R$ and use polynomial interpolation. 


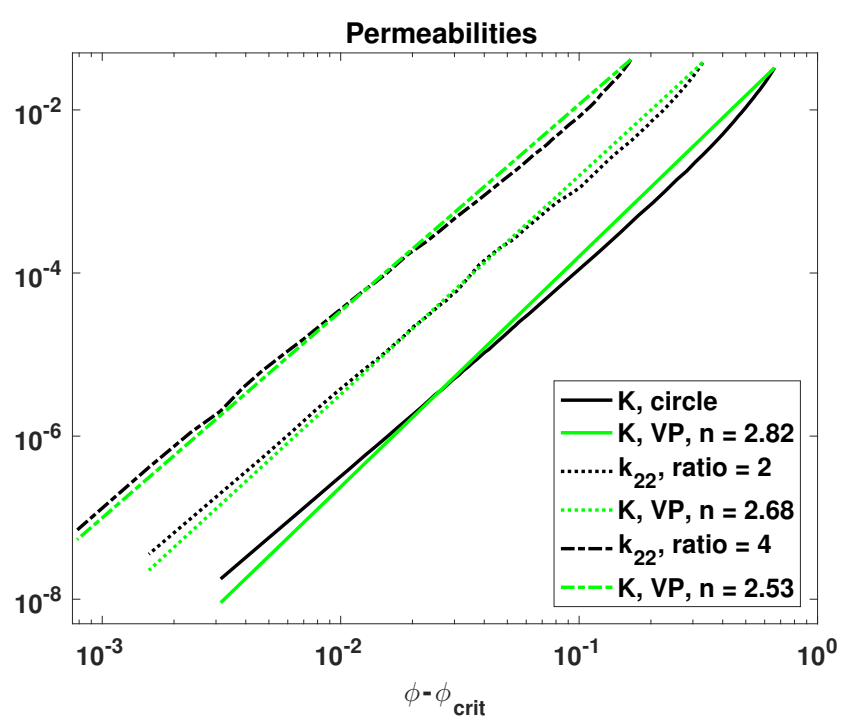

Fig. 12 Comparison of the permeability, or permeability in the $y_{2}$-direction when the porosity approaches the critical porosity. The green lines are the least-square fitted versions of equation (6) based on Verma and Pruess (1988).

\subsection{Approximate heat conductive cell problems}

The cell problem (3b) used to calculate the heat conductivity coefficients $\mathcal{A}_{f}$ and $\mathcal{A}_{g}$ can be approximated by solving a related cell problem analytically. The cell problem is formulated through the unknown functions $\Theta_{f}\left(y_{1}, y_{1}\right)$ and $\Theta_{g}\left(y_{1}, y_{2}\right)$, that should fulfill equation ( $3 \mathrm{~b})$ together with periodicity across the external cell boundaries:

$$
\Theta_{f}\left(y_{1}=-1 / 2\right)=\Theta_{f}\left(y_{1}=1 / 2\right), \Theta_{f}\left(y_{2}=-1 / 2\right)=\Theta_{f}\left(y_{2}=1 / 2\right)
$$

Our appraoch involves using polar coordinates and separation of variables; hence, we assume the solutions can be written on the form

$$
\Theta_{f}(r, \theta)=F_{r}(r) F_{\theta}(\theta), \Theta_{g}(r, \theta)=G_{r}(r) G_{\theta}(\theta) .
$$

However, as shown in the Appendix, our assumption of separation of variables together with the resulting solution form in the azimuthal direction will lead to the periodicity requirement on the external boundary not being fulfilled. Instead, we search an approximate solution through alternative boundary conditions for the external boundary. We consider two alternatives: Either dropping the external boundary, allowing $\Theta_{f}$ to be defined for all $r>R$; or, keeping the boundary, but use other means to obtain a unique solution. Redefining the cell problem, makes it more similar to the conductive single-inclusion solutions handled by Torquato (2013). Torquato (2013) also shows how to expand these solutions to effective medium approximations of multiphase media. However, the two approaches lead 


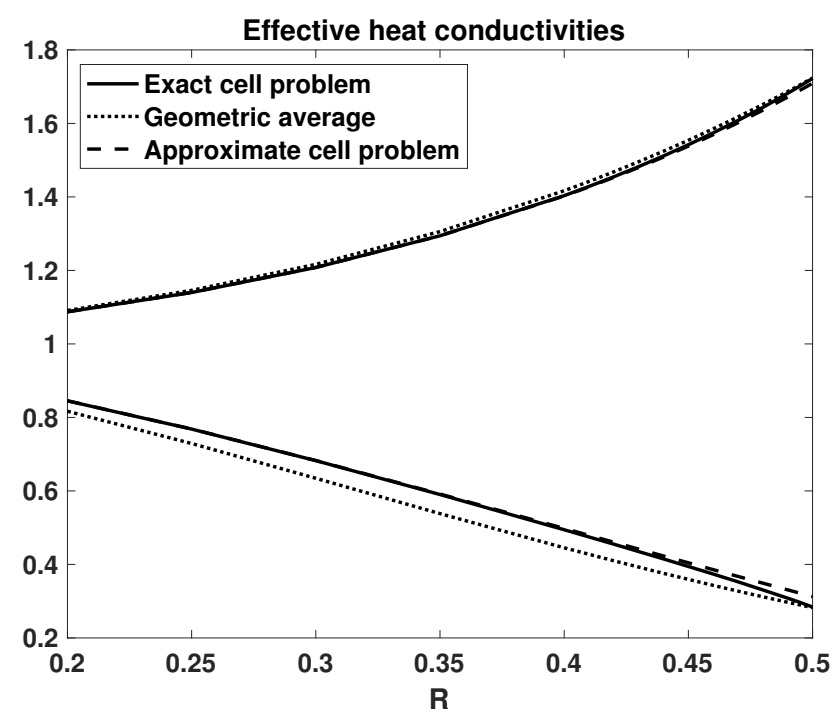

Fig. 13 Comparison of the effective heat conductivities. The three lower curves are for $\kappa_{f}=$ $1, \kappa_{g}=0.2$, while the three upper curves are for $\kappa_{f}=1, \kappa_{g}=2$.

to the same solution, namely:

$$
\begin{aligned}
& \mathcal{A}_{f}=\left(1-\pi R^{2}\right) \frac{1+\kappa}{1+\kappa+\pi R^{2}(1-\kappa)}, \\
& \mathcal{A}_{g}=\pi R^{2} \frac{2}{1+\kappa+\pi R^{2}(1-\kappa)} .
\end{aligned}
$$

The full derivation of the solution using the two approaches are found in the Appendix. Figure 13 compares the approximate effective heat conductivity from equation (7) with the exact solution found from the cell problem (3b) and the volume weighted geometric average $(5 \mathrm{~b})$. The approximate cell problem does a better job than the volume averaging except for large values of $R$.

\subsection{Approximate polynomials for the cell problem solutions}

We here try to estimate the effective quantities $\mathcal{A}_{u}(R), \mathcal{A}_{f}(R), \mathcal{A}_{g}(R)$ and $\mathcal{K}(R)$ with approximate polynomials based on numerically found solutions of the cell problems (3), which have been obtained for discrete values of $R$ within our interval of interest: $R \in[0.2,0.5]$. As van Noorden (2009a), we use a polynomial with powers of $R^{0}, R^{1 / 2}, R^{1}, R^{3 / 2}$ and $R^{2}$ for the ion diffusivity coefficient and the heat conductivity coefficients. While van Noorden (2009a) could use that the polynomial for $\mathcal{A}_{u}$ was 1 when $R=0$ and 0 when $R=0.5$, the latter is not the case for $\mathcal{A}_{f}$ as there is still a large amount of conductive heat transfer in the fluid when the porous medium is clogged. However, the polynomial for $\mathcal{A}_{g}$ would be 0 for $R=0$, and the polynomials approximating $\mathcal{A}_{f}$ and $\mathcal{A}_{g}$ should still fulfill that their sum is 1 for all $R$. 
The polynomial found to approximate $\mathcal{A}_{u}$ best in the least-square sense is

$\mathcal{A}_{u}(R) \approx 0.7232(0.5-R)^{1 / 2}-0.2166(0.5-R)+4.8785(0.5-R)^{3 / 2}-3.9439(0.5-R)^{2}$.

The coefficients of this polynomial are quite different than the ones found by van Noorden (2009a), despite fitting within the same interval. The curves provide similar values. The maximal error in the fitting points was $10^{-3}$ for van Noorden (2009a), which is also the case here.

We approximate $\mathcal{A}_{f}$ and $\mathcal{A}_{g}$ with polynomials only for $\kappa=2$ and $\kappa=0.2$, to indicate the different behaviors for when $\kappa$ is larger/smaller than 1. For $\kappa=2$, the polynomials found to approximate $\mathcal{A}_{f}$ and $\mathcal{A}_{g}$ best in the least-square sense are

$$
\begin{aligned}
& \mathcal{A}_{f}(R) \approx 3.3481 R^{1 / 2}-19.5551 R+38.2452 R^{3 / 2}-27.33065 R^{2}+1 . \\
& \mathcal{A}_{g}(R) \approx-3.3433 R^{1 / 2}+19.5274 R-38.1923 R^{3 / 2}+27.2974 R^{2} .
\end{aligned}
$$

For $\kappa=0.2$, the polynomials found to approximate $\mathcal{A}_{f}$ and $\mathcal{A}_{g}$ best in the leastsquare sense are

$$
\begin{aligned}
& \mathcal{A}_{f}(R) \approx 1.0282 R^{1 / 2}-4.3256 R+3.8254 R^{3 / 2}-3.2395 R^{2}+1 . \\
& \mathcal{A}_{g}(R) \approx-1.0317 R^{1 / 2}+4.3455 R-3.8638 R^{3 / 2}+3.2639 R^{2} .
\end{aligned}
$$

The polynomials for $\mathcal{A}_{f}$ are assumed to have a constant factor of 1 as $\mathcal{A}_{f}(0)=$ 1 and $\mathcal{A}_{g}(0)=0$, but are otherwise only fitted within the interval of interest. Due to numerical roundoff error and as the least-square fitting have been done independently for the polynomials, the approximating polynomials do not sum up exactly to 1 for all $R$, but deviate from 1 with up to $10^{-4}$. The maximum error for the fitting points is $10^{-3}$ for the effective heat conductivities in both cases. Plotting the effective heat conductivity calculated from the cell problems together with the approximate effective heat conductivity from the above polynomials, reveals the two curves being virtually equal. The two cases of high and low $\kappa$ results in polynomials of similar structure, but the higher order coefficients are of relative higher importance for $R$-values close to 0.5 when $\kappa=2$, while the linear terms dominate for $\kappa=0.2$. This behavior is due to the more curved shapes of the high $\kappa$ curves near $R=0.5$ seen in Figure 3 .

The behavior of the permeability close to clogging is important to capture when modeling precipitation and clogging as the flow pattern can change significantly as pores close (and possibly reopen) and the relative permeability variations can vary within several orders of magnitude close to clogging. In van Noorden (2009a) the permeability was fitted with a sum of first, second and third order powers of $(0.5-R)$. Van Noorden did not focus on clogging and fitted with the factors that would capture the behavior over his whole region of interest. However, the sum of these three terms implies that the permeability should approach zero as $O(0.5-R)$ as the first order term will dominate the two others when $R$ approaches 0.5 . Investigating the permeability values when $R$ is close to 0.5 , as seen in the right part of Figure 6, reveals that the permeability does not approach zero linearly when close to clogging. The logarithmic plot indicates an inclination number of approximately $5 / 2$ for $R$ close to 0.5 . This means that to capture the behavior close to clogging, the approximating polynomial should not contain terms with $(0.5-R)$-factors of power less than $5 / 2$. The polynomial can however contain 
higher order terms as these will not dominate close to clogging. A polynomial that is found to approximate the permeability values satisfactory, both away from clogging and close to clogging, is

$$
\mathcal{K}(R) \approx 0.6191(0.5-R)^{5 / 2}-0.4892(0.5-R)^{3}+1.0441(0.5-R)^{7 / 2} .
$$

The maximum error in the fitting points is $10^{-4}$, and is found for low values of $R$. The maximum relative error is 0.08 , and occurs when the radius is close to clogging. Approximate polynomials with terms containing first order $(0.5-R)$-terms are found to have a relative error larger than 1 . An alternative approach to the above polynomial would be to approximate the behavior of the permeability with two polynomials; one valid for low values of $R$ and one valid for $R$ close to 0.5 , and tie these two polynomials together with continuity constraints in some breaking point. This approach could however cause issues in a numerical implementation. As the logarithmic plot in Figure 6 does not indicate any obvious breaking points, using the same polynomial for the whole fitting interval $[0.2,0.5)$ should be feasible in this case.

\section{Case study: clogging}

To investigate the behavior of the upscaled effective equations (4) in a numerical implementation, we create a case study with initial and boundary conditions, mineral solubilities and rock properties such that clogging will occur at some distance away from the well. The Darcy scale domain is assumed to be filled with microscopic pores as Figure 1 next to each other, in line with the periodic assumption in deriving the upscaled model in Bringedal et al (2016). The microscopic cells are not explicitly visible in the Darcy scale domain, as they in the homogenization limit are assumed infinitesimally small, but contribute through the cell problem solution for effective quantities, e.g. permeability, at each macroscopic point in the domain. The goal of this case study is to see how the upscaled model equations behave close to clogging and how the heat transport is affected by the clogging.

\subsection{Case study formulation and implementation}

Note that all parameters and numbers specified in the following are non-dimensional according to the non-dimensionalization given in Bringedal et al (2016). Temperatures are also shifted to lie between 0 and 1 . The macroscale domain is a twodimensional square $x \in[0,1]^{2}$ with an injection and production well operating at constant pressure. The injection well is located in $\left(x_{1}, x_{2}\right)=(0.2,0.2)$ and the production well in $\left(x_{1}, x_{2}\right)=(0.8,0.8)$. External boundaries are impermeable and insulated. The domain is initialized with a temperature of 1 and with an ion concentration such that the fluid is fully saturated with ions; in this case a concentration of $\sqrt{0.75}$. We mimic a reservoir with two types of rock that are such that the temperature drop due to injecting fluid of temperature 0 triggers mineral dissolution near the injection well and mineral precipitation further away. The two types of rocks meet along the line $x_{1}=0.5$. The injected fluid has the same ion concentration as the initial to resemble reinjection of produced fluid, but mineral dissolution will occur near the injection well due to the temperature drop. As 
fluid flows towards the production well, minerals start precipitating when crossing $x_{1}=0.5$, both due to the incoming fluid having a larger ion concentration from the upstream mineral dissolution, but also caused by the gradual temperature decrease.

The Darcy scale model equations (4) are modeled fully coupled with Euler forward in time and the control volume method on a square grid with two point flux approximation in space. The injection wells are handled through a mass conservative implementation as described in Bringedal et al (2014). As clogging is a difficult process to model due to the degenerate nature of the transport equations, we use a time step constraint when one or more cells are close to clogging. Also, we consider a cell clogged when the grain radius exceeds $R=0.4997$. When a cell clogs, pressure is undefined in this cell and is no longer solved for. Heat conduction and chemical reactions are still solved for in the clogged cells, the latter to allow for any reopening at a later time. The dissolution rate, which has a discontinuity from $(1)$, is regularized. We mention there are more sophisticated ways of dealing with both mineral dissolution (containing a discontinuous reaction term) and precipitation possibly leading to clogging, and refer to Agosti et al $(2015 \mathrm{a}, \mathrm{b})$ for details on event-driven implementation of this aspect. Despite using Euler forward for time stepping, the resulting system of equations is nonlinear due to the nonlinearities in the time derivatives. The nonlinear system of equations is solved with Newton iterations, using the solution from the previous time step as initial guess.

The effective quantities $\mathcal{A}_{u}(R), \mathcal{A}_{f}(R), \mathcal{A}_{g}(R)$ and $\mathcal{K}(R)$ arising from the cell problems are implemented with their approximating polynomials found in Section 4.2. Hence, the cell problems do not need to be solved at each mesh point at each time step, but pore scale effects are included through the approximate polynomials of the effective quantities arising from cell problem solutions. We have used $\kappa_{f}=1$ and $\kappa_{g}=2$, which is a representative correspondence between heat conductivity in fluid and grain for many soils/rocks. The porous medium is initialized with $R=0.3$ and the amount of minerals that cannot dissolve is chosen to correspond to $R_{\min }=0.2$. Note that this choice of homogeneous initial condition (and minimum radius) for the rock is not a necessity for the model formulation and only chosen for convenience. Other parameters appearing in the model equations (4) are given by

$$
\begin{gathered}
D=1, \quad \varsigma=1, \quad k=1, \quad \alpha=1, \quad \rho=1, \\
\beta_{\rho}=0.01, \quad \beta_{\mu}=0.01, \quad \rho_{0}=2, \quad \mu_{0}=1,
\end{gathered}
$$

where the fluid density is $\rho_{f}(T)=\rho_{0}-\beta_{\rho} T$ and viscosity is $\mu(T)=\mu_{0}-\beta_{\mu} T$. The injection pressure is 100 , and production pressure is 0.5 , which induces a pressure difference in the domain giving flow rates around 1 initially, hence advective and diffusive processes occur at about the same time scale, which is one of the assumptions in the derivation of the upscaled model by Bringedal et al (2016).

\subsection{Numerical results}

At injection start the fluid flows towards the production well, bringing along lower temperatures and, as the minerals close to the injection well starts dissolving, larger ion concentrations. Upon reaching the other rock type at $x_{1}=0.5$ minerals 


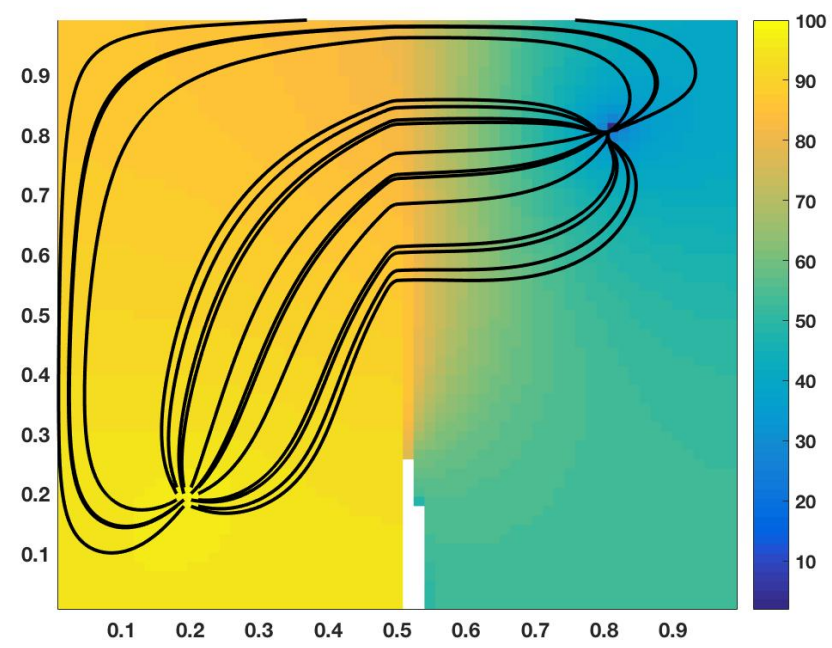

Fig. 14 Pressure distribution (colors) 1 time unit after injection start. Clogged cells are marked as white as the pressure is undefined. Black lines are (instantaneous) stream lines. The flow trajectories are not smooth across $x_{1}=0.5$ as the grain radius $R$ and hence also permeability $\mathcal{K}$ are discontinuous here.

precipitate, resulting in gradually lower porosities and permeabilities behind this line. Also, as the minerals dissolves close to the injection well, the effective heat conductivity decreases due to $\kappa_{f}$ being lower than $\kappa_{g}$. Although one would expect clogging to first occur in $\left(x_{1}, x_{2}\right)=(0.5,0.2)$, this being the point closest to the injection well where clogging can occur, the clogging first occurs at $\left(x_{1}, x_{2}\right)=$ $(0.5,0)$. This is caused by the point $\left(x_{1}, x_{2}\right)=(0.5,0)$ being cooled faster than $\left(x_{1}, x_{2}\right)=(0.5,0.2)$, despite being further away from the injection point, triggering larger precipitation rates. The differences in cooling (and initiating of clogging) are small, but this faster cooling in $(0.5,0)$ is a result of the heat conductivity being larger in regions where less minerals have dissolved. The convective cooling is (initially) focused mainly along the band between injection and production well, hence conductive cooling dominates between the injection well and the line $x_{1}=0.5$ for low $x_{2}$-values. As the dissolving occurs slower along $x_{2}=0$ the heat conduction is faster than for $x_{2}=0.2$. The fact that clogging occurs first at $\left(x_{1}, x_{2}\right)=(0.5,0)$ in stead of in $(0.5,0.2)$ is a nice example of the effect of the interplay between heat transfer and reactive transport. Clogging gradually occurs along increased values of $x_{2}$ as these cells are being cooled, and at the same time the flow is forced to follow trajectories further away from the clogged region. Due to heat conduction still playing a role in the blocked region, clogging still occurs behind the line $x_{1}=0.5$. Figure 14 shows the pressure distribution and some flow trajectories some time after clogging has started near $\left(x_{1}, x_{2}\right)=(0.5,0)$.

There is still heat conduction in the clogged cells. In fact, the clogged cells encounter an increased effective heat conductivity as the grain heat conductivity is larger than fluid heat conductivity. Hence, the temperature distribution is not strongly affected by the clogged flow paths in this case study. Figure 15 shows how 

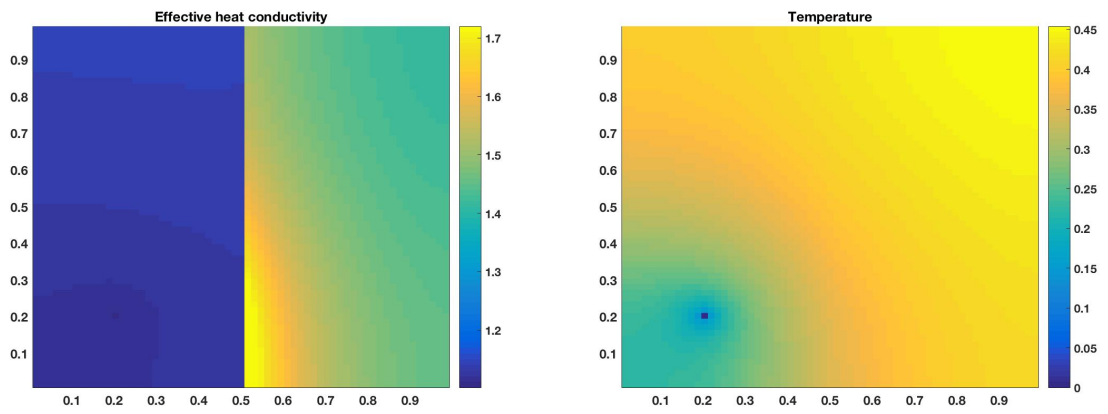

Fig. 15 Effective heat conductivity distribution (left) and temperature distribution (right) 1 time unit after injection start. Temperature is still well defined in the clogged cell, and is where the highest effective heat conductivity values are found. The increased heat conductivity is the reason for the relatively smooth temperature field despite flow paths being clogged.

the effective heat conductivity and temperature distributions are at the same time as the pressure distribution in Figure 14.

\section{Summary and conclusion}

In this paper we have shown how the effective behavior of heat conductivity and permeability performs in a porous medium where porosity changes due to mineral precipitation and dissolution. The heat conductivity coefficients and permeability is calculated through cell problems derived from a pore scale formulation based on Bringedal et al (2016), using circular and elliptic grains as illustrative examples. We have shown how the effective behavior for the heat conductivity differs from the usually applied porosity-weighted averaging between fluid and grain, where the geometric averaging performs better in the isotropic case and harmonic, geometric or arithmetic averaging is the better approximation depending on degree of and direction of anisotropy.

We have solved the heat conductivity cell problems numerically, but approximate versions can be solved analytically. The resulting solutions for circular grains capture the effective behavior well and generally better than the geometric averaging. Hence, for practical use in simulators, one can either apply the approximate analytical solutions, or approximate polynomials based on least square fitting of discretely solved cell problems. When doing least square fitting for the cell problems one has to decide which order terms to include in the polynomial, and this can potentially alter the behavior of the resulting effective parameters, especially near critical points as clogging represents. As the permeability can vary with several orders of magnitude near clogging, approximating with suitable terms and factors are especially important in this case.

As we are particularly interested in the behavior of the effective model near clogging, a case study where clogging would occur was designed. The case study mimics a geothermal reservoir where cold fluid is injected, and where composition of different rock types leads to mineral precipitation at some distance away from the well. The reduced permeability and gradual clogging of the medium lead to 
the injected water being forced through new flow paths further away from the production well, and at the same time increasing the effective heat conductivity through the medium.

\section{Appendix}

Section 4.1 dealt with analytical solutions of an approximate heat conductive cell problem and we here give the derivation of these solutions. We seek the unknown functions $\Theta_{f}\left(y_{1}, y_{1}\right)$ and $\Theta_{g}\left(y_{1}, y_{2}\right)$, that should fulfill equation (3b) together with periodicity across the external cell boundary:

$$
\Theta_{f}\left(y_{1}=-1 / 2\right)=\Theta_{f}\left(y_{1}=1 / 2\right), \Theta_{f}\left(y_{2}=-1 / 2\right)=\Theta_{f}\left(y_{2}=1 / 2\right)
$$

We use polar coordinates and assume separation of variables; hence, the solutions can be written

$$
\Theta_{f}(r, \theta)=F_{r}(r) F_{\theta}(\theta), \Theta_{g}(r, \theta)=G_{r}(r) G_{\theta}(\theta) .
$$

Then, the model equations from $(3 \mathrm{~b})$ are

$$
\begin{aligned}
\frac{1}{r} \frac{d}{d r}\left(r \frac{d F_{r}}{d r}\right) F_{\theta}+\frac{1}{r^{2}} \frac{d^{2} F_{\theta}}{d \theta^{2}} F_{r} & =0, y \in Y_{0}(x, t) \\
\frac{1}{r} \frac{d}{d r}\left(r \frac{d G_{r}}{d r}\right) G_{\theta}+\frac{1}{r^{2}} \frac{d^{2} G_{\theta}}{d \theta^{2}} G_{r} & =0, y \in G_{0}(x, t),
\end{aligned}
$$

while the interior boundary conditions can be written as

$$
\begin{aligned}
\cos \theta+F_{r}^{\prime}(R) F_{\theta} & =\kappa \cos \theta+\kappa G_{r}^{\prime}(R) G_{\theta} \text { at } r=R, \\
F_{r}(R) F_{\theta} & =G_{r}(R) G_{\theta} \text { at } r=R,
\end{aligned}
$$

and for all $\theta$. The model equations for $F_{\theta}$ and $G_{\theta}$ reduces to $F_{\theta}^{\prime \prime}=-\lambda F_{\theta}$ for some number $\lambda$, which, together with the interior boundary condition (8a), suggest that

$$
F_{\theta}(\theta)=G_{\theta}(\theta)=\cos \theta
$$

The model equations for $F_{r}$ and $G_{r}$ are then reduced to $r^{2} F_{r}^{\prime \prime}+r F_{r}^{\prime}-F_{r}=0$, which have the general solutions

$$
F_{r}(r)=b_{1} r+b_{2} \frac{1}{r}, G_{r}(r)=b_{3} r+b_{4} \frac{1}{r},
$$

where $b_{1}, b_{2}, b_{3}, b_{4}$ are integration constants. However, our assumption of separation of variables together with the solution in $\theta$, leads to the periodicity requirement on the external boundary not being fulfilled. There is periodicity across the horizontal boundaries, but periodicity across the vertical boundaries are not met. We instead search an approximate solution through alternative boundary conditions for the external boundary and consider two approaches: Either dropping the external boundary and allowing $\Theta_{f}$ to be well-defined for all $r>R$; or, keeping the boundary, but neglect the boundary conditions and use other means to determine the constants $b_{1}, b_{2}, b_{3}, b_{4}$. 
Alterative I: Infinite domain

We allow $\Theta_{f}$ to be defined for all $r>R$, hence we require it to be finite as $r \rightarrow \infty$. Also, as $\Theta_{g}$ must be well-defined as $r \rightarrow 0$, the general solutions in $r$ are

$$
F_{r}(r)=b_{2} \frac{1}{r}, G_{r}(r)=b_{3} r
$$

Applying the internal boundary conditions (8) results in $b_{2}=R^{2}(1-\kappa) /(1+\kappa)$ and $b_{3}=(1-\kappa) /(1+\kappa)$. Hence, the solutions of the approximate cell problem are

$$
\begin{aligned}
& \Theta_{f}(r, \theta)=R^{2} \frac{1-\kappa}{1+\kappa} \frac{1}{r} \cos \theta, \\
& \Theta_{g}(r, \theta)=\frac{1-\kappa}{1+\kappa} r \cos \theta .
\end{aligned}
$$

The approximate heat conductivity coefficients are then

$$
\begin{aligned}
& \mathcal{A}_{f}=\int_{Y_{0}(x, t)}\left(1+\partial_{x} \Theta_{f}\right) d y=1-\pi R^{2}, \\
& \mathcal{A}_{g}=\int_{G_{0}(x, t)}\left(1+\partial_{x} \Theta_{g}\right) d y=\pi R^{2} \frac{2}{1+\kappa} .
\end{aligned}
$$

Although $\Theta_{f}$ is now defined for all $r>R$, the original integration area for $\mathcal{A}_{f}$ is used. As we made an error by allowing $\Theta_{f}$ to exist as $r \rightarrow \infty$, and as we know the sum of $\mathcal{A}_{f}$ and $\mathcal{A}_{g}$ should be 1 when in the finite pore space domain, our above approximation can be improved by scaling the above coefficients with the same number such that their sum is 1 . Hence,

$$
\begin{aligned}
& \mathcal{A}_{f}=\left(1-\pi R^{2}\right) \frac{1+\kappa}{(1+\kappa)\left(1-\pi R^{2}\right)+2 \pi R^{2}} \\
& \mathcal{A}_{g}=\pi R^{2} \frac{2}{(1+\kappa)\left(1-\pi R^{2}\right)+2 \pi R^{2}} .
\end{aligned}
$$

Alternative II: No periodic boundary condition

In this approach we keep the external boundary but do not require periodicity across it. We still require $\Theta_{g}$ to be well-defined as $r \rightarrow 0$, hence $b_{4}=0$ and the three remaining constants must be such that they fulfill the two internal boundary conditions (8):

$$
\begin{aligned}
& 1+b_{1}-\frac{b_{2}}{R^{2}}=\kappa+\kappa b_{3}, \\
& 1+b_{1} R+\frac{b_{2}}{R}=b_{3} R .
\end{aligned}
$$

Expressing $b_{2}, b_{3}$ as functions of $b_{1}$ leads to

$$
\begin{aligned}
& b_{2}=R^{2} \frac{1-\kappa}{1+\kappa}\left(1+b_{1}\right), \\
& b_{3}=\frac{1-\kappa}{1+\kappa}+\frac{2 b_{1}}{1+\kappa} .
\end{aligned}
$$


The solutions of the (approximate) cell problem are then

$$
\begin{aligned}
& \Theta_{f}(r, \theta)=\left(b_{1}+\frac{R^{2}}{r^{2}} \frac{1-\kappa}{1+\kappa}\left(1+b_{1}\right)\right) r \cos \theta \\
& \Theta_{g}(r, \theta)=\left(\frac{1-\kappa}{1+\kappa}+\frac{2 b_{1}}{1+\kappa}\right) r \cos \theta
\end{aligned}
$$

where the constant $b_{1}$ is to be determined later. The heat conductivities are then

$$
\begin{aligned}
& \mathcal{A}_{f}=\int_{Y_{0}(x, t)}\left(1+\partial_{x} \Theta_{f}\right) d y=\left(1-\pi R^{2}\right)\left(1+b_{1}\right), \\
& \mathcal{A}_{g}=\int_{G_{0}(x, t)}\left(1+\partial_{x} \Theta_{g}\right) d y=\pi R^{2} \frac{2\left(1+b_{1}\right)}{1+\kappa}
\end{aligned}
$$

We now require the sum $\mathcal{A}_{f}+\mathcal{A}_{g}$ to be 1 , and use this to determine $b_{1}$. This way,

$$
\begin{aligned}
& \mathcal{A}_{f}=\left(1-\pi R^{2}\right) \frac{1+\kappa}{1+\kappa+\pi R^{2}(1-\kappa)}, \\
& \mathcal{A}_{g}=\pi R^{2} \frac{2}{1+\kappa+\pi R^{2}(1-\kappa)} .
\end{aligned}
$$

Although this derivation uses different assumptions than Alternative I, the resulting approximate heat conductivity coefficients $\mathcal{A}_{f}$ and $\mathcal{A}_{g}$ found in (9) and (10) are identical.

Acknowledgements K. Kumar acknowledges the support by the Research Council of Norway under the project IMMENS no. 255426 and "EOR upscaling - from lab to field", Petromaks 2 project. The authors would like to thank Nadja Ray and Raphael Schulz from FAU Erlangen-Nürnberg for their useful discussions supported by the Research Council of Norway (RCN) and German Academic Exchange Service (DAAD) project No. 255773, "Upscaling Evolving Microstructures and its Applications".

\section{References}

Agosti, A., Formaggia, L., Scotti, A.: Analysis of a model for precipitation and dissolution coupled with a Darcy flux, Journal of mathematical analysis and applications 431:752 781 (2015a). DOI 10.1016/j.jmaa.2015.06.003

Agosti, A., Formaggia, L., Giovanardi, B., Scotti, A.: MOX-Report No. 08/2015, Numerical simulation of geochemical compaction with discontinuous reactions. Tech. rep., MOX, Politecnico di Milano (2015b)

Bringedal, C., Berre, I., Radu, F.A.: An approach for investigation of geochemical rock-fluid interactions. In: Proceedings, Thirty-Ninth Workshop on Geothermal Reservoir Engineering, Stanford University (2014)

Bringedal, C., Berre, I., Pop, I.S., Radu, F.A.: A model for non-isothermal flow and mineral precipitation and dissolution in a thin strip. Journal of Computational and Applied Mathematics 289:346 - 355 (2015). DOI http://dx.doi.org/10.1016/j.cam.2014.12.009

Bringedal, C., Berre, I., Pop, I.S., Radu, F.A.: Upscaling of Non-isothermal Reactive Porous Media Flow with Changing Porosity. Transport in Porous Media 114(2):371-393 (2016). DOI 10.1007/s11242-015-0530-9

Chadam, J., Hoff, D., Merino, E., Ortoleva, P., Sen, A.: Reactive infiltration instabilities. IMA Journal of Applied Mathematics, 36(3):207-221 (1986)

Chadam, J., Peirce, A., Ortoleva, P.: Stability of reactive flows in porous media: coupled porosity and viscosity changes. SIAM Journal on Applied Mathematics, 51(3):684-692 (1991) 
COMSOL Inc. Version 4.2, http://www.comsol.com, (2011)

van Duijn, C.J., Knabner, P.: Travelling wave behavior of crystal dissolution in porous media flow. European Journal of Applied Mathematics 8:49-72 (1997)

van Duijn, C.J., Pop, I.S.: Crystal dissolution and precipitation in porous media: pore scale analysis. Journal für die reine und angewandte Matematik 577:171-211 (2004)

Duval, F., Fichot, F., Quintard, M.: A local thermal non-equilibrium model for two-phase flows with phase-change in porous media. International Journal of Heat and Mass Transfer 47(3):613-639 (2004)

Frank, F.: Numerical studies of models for elektrokinetic flow and charged solute transport in periodic porous media. $\mathrm{PhD}$ thesis, Friedrich-Alexander-Universität Erlangen-Nürnberg (FAU) (2013)

Frank, F., Ray, N., Knabner, P.: Numerical investigation of homogenized Stokes-Nernst-PlanckPoisson systems. Computing and visualization in science 14(8): 385-400 (2011)

Knabner, P., van Duijn, C.J., Hengst, S.: An analysis of crystal dissolution fronts in flows through porous media. Part 1: Compatible boundary conditions. Advances in water resources 18(3):171-185 (1995)

Kumar, K., Neuss-Radu, M., Pop, I.S.: Homogenization of a pore scale model for precipitation and dissolution in porous media. IMA Journal of Applied Mathematics, 81(5), 877-897 (2016)

Kumar, K., van Noorden, T.L., Pop, I.S.: Effective dispersion equations for reactive flows involving free boundaries at the microscale. Multiscale Modeling \& Simulation 9(1):29-58 (2011)

Kumar, K., Pop, I.S., Radu, F.A.: Convergence analysis of mixed numerical schemes for reactive flow in a porous medium. SIAM Journal on Numerical Analysis 51(4):2283-2308 (2013)

Kumar, K., Pop, I.S., Radu, F.A.: Convergence analysis for a conformal discretization of a model for precipitation and dissolution in porous media. Numerische Mathematik, 127(4), 715-749 (2014)

Kumar, K., Wheeler, M.F., Wick, T.: Reactive flow and reaction-induced boundary movement in a thin channel. SIAM Journal on Scientific Computing, 35(6), B1235-B1266 (2013)

Libbey, R.B., Williams-Jones, A.E.: Relating sulfide mineral zonation and trace element chemistry to subsurface processes in the Reykjanes geothermal system, Iceland. Journal of Volcanology and Geothermal Research 310:225-241 (2016)

McNamara, D.D., Lister, A., Prior, A.J.: Calcite sealing in a fractured geothermal reservoir: Insights from combined EBSD and chemistry mapping. Journal of Volcanology and Geothermal Research 323:38-52 (2016)

Mielke, P., Nehler, M., Bignall, G., Sass, I.: Thermo-physical rock properties and the impact of advancing hydrothermal alteration - A case study from the Tauhara geothermal field, New Zealand. Journal of Volcanology and Geothermal Research 301:14-28 (2015)

Mroczek, E.K., White, S.P., Graham, D.J.: Deposition of amorphous silica in porous packed beds predicting the lifetime of reinjection aquifers. Geothermics 29(6):737-757 (2000)

Nield, D.A., Bejan, A.: Convection in Porous Media, 4th edition. Springer Science \& Business Media (2013)

van Noorden, T.L.: Crystal precipitation and dissolution in a porous medium: effective equations and numerical experiments. Multiscale Modeling \& Simulation 7(3):1220-1236 (2009a)

van Noorden, T.L.: Crystal precipitation and dissolution in a thin strip. European Journal of Applied Mathematics 20:69-91 (2009b)

van Noorden, T.L., Pop, I.S., Röger, M.: Crystal dissolution and precipitation in porous media: L1-contraction and uniqueness. In: Discrete Contin. Dyn. Syst.,(Dynamical Systems and Differential Equations. Proceedings of the 6th AIMS International Conference, suppl.), pp 1013-1020 (2007)

van Noorden, T.L., Pop, I.S., Ebigbo, A., Helmig, R.: An upscaled model for biofilm growth in a thin strip. Water Resources Research 46(6) (2010)

Pape, H., Clauser, C., Iffland, J., Krug, R., Wagner, R.: Anhydrite cementation and compaction in geothermal reservoirs: Interaction of pore-space structure with flow, transport, p-t conditions, and chemical reactions. International Journal of Rock Mechanics and Mining Sciences 42(7):1056-1069 (2005)

Peter, M.A.: Coupled reaction-diffusion processes inducing an evolution of the microstructure: Analysis and homogenization. Nonlinear Analysis: Theory, Methods \& Applications 70(2):806-821 (2009) 
Ray, N., van Noorden, T.L., Radu, F.A., Friess, W., Knabner, P.: Drug release from collagen matrices including an evolving microstructure. ZAMM-Journal of Applied Mathematics and Mechanics 93(10-11):811-822 (2013)

Schulz, R., Ray, N., Frank, F., Mahalo, H.S., Knabner, P.: Strong solvability up to clogging of an effective diffusion-precipitation model in an evolving porous medium. European Journal of Applied Mathematics 1-29 (2016)

Sonnenthal, E., Ito, A., Spycher, N., Yui, M., Apps, J., Sugita, Y., Conrad, M., Kawakami, S.: Approaches to modeling coupled thermal, hydrological, and chemical processes in the drift scale heater test at yucca mountain. International Journal of Rock Mechanics and Mining Sciences 42(5):698-719 (2005)

Taron, J., Elsworth, D.: Thermal-hydrologic-mechanical-chemical processes in the evolution of engineered geothermal reservoirs. International Journal of Rock Mechanics and Mining Sciences 46:855-654 (2009)

Torquato, S.: Random heterogeneous materials: microstructure and macroscopic properties. Springer Science \& Business Media (2013)

Verma, A., Pruess, K.: Thermohydrological conditions and silica redistribution near highlevel nuclear wastes emplaced in saturated geological formations. Journal of Geophysical Research: Solid Earth (1978-2012) 93(B2):1159-1173 (1988)

Wagner, R., Kühn, M., Meyn, V., Pape, H., Vath, U., Clauser, C.: Numerical simulation of pore space clogging in geothermal reservoirs by precipitation of anhydrite. International Journal of Rock Mechanics and Mining Sciences 42(7):1070-1081 (2005)

White, S.P., Mroczek, E.K.: Permeability changes during the evolution of a geothermal field due to the dissolution and precipitation of quartz. Transport in porous media 33(1-2):81101 (1998)

Woodside, W.M.J.H., Messmer, J.H.: Thermal Conductivity of Porous Media. I. Unconsolidated sands. Journal of Applied Physics 32:1688-1699 (1961)

Xu, T., Rose, P., Fayer, S., Pruess, K.: On modeling of chemical stimulation of an enhanced geothermal system using a high pH solution with chelating agent. Geofluids 9:167-177 (2009)

Xu, T., Spycher, N., Sonnenthal, E., Zheng, L., Pruess, K.: TOUGHREACT user's guide: A simulation program for non-isothermal multiphase reactive transport in variably saturated geologic media, version 2.0. Earth Sciences Division, Lawrence Berkeley National Laboratory, Berkeley, USA (2012)

Zhao, C.: Physical and chemical dissolution front instability in porous media: theoretical analyses and computational simulations. Springer (2014) 


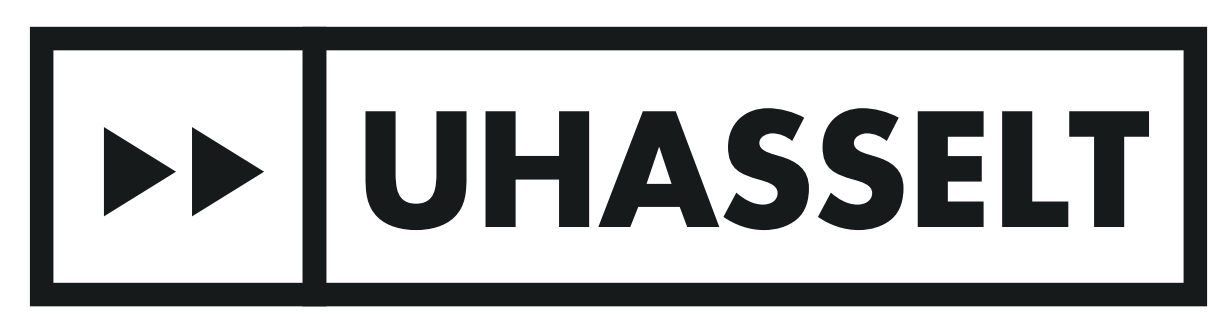

\section{UHasselt Computational Mathematics Preprint Series}

\section{7}

UP-17-10 Carina Bringedal and Kundan Kumar, Effective behavior near clogging in upscaled equations for non-isothermal reactive porous media flow, 2017

UP-17-09 Alexander Jaust, Balthasar Reuter, Vadym Aizinger, Jochen Schütz and Peter Knabner, FESTUNG: A MATLAB / GNU Octave toolbox for the discontinuous Galerkin method. Part III: Hybridized discontinuous Galerkin (HDG) formulation, 2017

UP-17-08 David Seus, Koondanibha Mitra, Iuliu Sorin Pop, Florin Adrian Radu and Christian Rohde, A linear domain decomposition method for partially saturated flow in porous media, 2017

UP-17-07 Klaus Kaiser and Jochen Schütz, Asymptotic Error Analysis of an IMEX Runge-Kutta method, 2017

UP-17-06 Hans van Duijn, Koondanibha Mitra and Iuliu Sorin Pop, Travelling wave solutions for the Richards equation incorporating nonequilibrium effects in the capillarity pressure, 2017

UP-17-05 Hans van Duijn and Koondanibha Mitra, Hysteresis and Horizontal Redistribution in Porous Media, 2017

UP-17-04 Jonas Zeifang, Klaus Kaiser, Andrea Beck, Jochen Schütz and ClausDieter Munz, Efficient high-order discontinuous Galerkin computations of low Mach number flows, 2017

UP-17-03 Maikel Bosschaert, Sebastiaan Janssens and Yuri Kuznetsov, Switching to nonhyperbolic cycles from codim-2 bifurcations of equilibria in DDEs, 2017

UP-17-02 Jochen Schütz, David C. Seal and Alexander Jaust, Implicit multiderivative collocation solvers for linear partial differential equations with discontinuous Galerkin spatial discretizations, 2017 
UP-17-01 Alexander Jaust and Jochen Schütz, General linear methods for time-dependent PDEs, 2017

\section{6}

UP-16-06 Klaus Kaiser and Jochen Schütz, A high-order method for weakly compressible flows, 2016

UP-16-05 Stefan Karpinski, Iuliu Sorin Pop, Florin A. Radu, A hierarchical scale separation approach for the hybridized discontinuous Galerkin method, 2016

UP-16-04 Florin A. Radu, Kundan Kumar, Jan Martin Nordbotten, Iuliu Sorin $P o p$, Analysis of a linearization scheme for an interior penalty discontinuous Galerkin method for two phase flow in porous media with dynamic capillarity effects , 2016

UP-16-03 Sergey Alyaev, Eirik Keilegavlen, Jan Martin Nordbotten, Iuliu Sorin Pop, Fractal structures in freezing brine, 2016

UP-16-02 Klaus Kaiser, Jochen Schütz, Ruth Schöbel and Sebastian Noelle, A new stable splitting for the isentropic Euler equations, 2016

UP-16-01 Jochen Schütz and Vadym Aizinger, A hierarchical scale separation approach for the hybridized discontinuous Galerkin method, 2016

All rights reserved. 